\title{
An Angiotensin-Responsive Connection from the Lamina Terminalis to the Paraventricular Nucleus of the Hypothalamus Evokes Vasopressin Secretion to Increase Blood Pressure in Mice
}

\author{
Charles J. Frazier, ${ }^{1,2}$ Scott W. Harden, ${ }^{1}$ Amy R. Alleyne, ${ }^{1}$ Mazher Mohammed, ${ }^{1}$ Wanhui Sheng, ${ }^{1}$ \\ Justin A. Smith, ${ }^{1}$ Khalid Elsaafien, ${ }^{1}$ Eliot A. Spector, ${ }^{1}$ Dominique N. Johnson, ${ }^{4}$ Karen A. Scott, ${ }^{1}$ \\ Eric G. Krause, ${ }^{1,2,3}$ and Annette D. de Kloet ${ }^{2,3,4}$ \\ ${ }^{1}$ Department of Pharmacodynamics, College of Pharmacy, University of Florida, Gainesville, Florida 32611 , ${ }^{2}$ Center for Integrative Cardiovascular and \\ Metabolic Diseases, University of Florida, Gainesville, Florida 32611, ${ }^{3}$ Evelyn F. and William L. McKnight Brain Institute, University of Florida, Gainesville, \\ Florida 32611, and ${ }^{4}$ Department of Physiology and Functional Genomics, College of Medicine, University of Florida, Gainesville, Florida 32611
}

Blood pressure is controlled by endocrine, autonomic, and behavioral responses that maintain blood volume and perfusion pressure at levels optimal for survival. Although it is clear that central angiotensin type 1a receptors (ATlaR; encoded by the Agtr1a gene) influence these processes, the neuronal circuits mediating these effects are incompletely understood. The present studies characterize the structure and function of ATlaR neurons in the lamina terminalis (containing the median preoptic nucleus and organum vasculosum of the lamina terminalis), thereby evaluating their roles in blood pressure control. Using male Agtrla-Cre mice, neuroanatomical studies reveal that ATlaR neurons in the area are largely glutamatergic and send projections to the paraventricular nucleus of the hypothalamus (PVN) that appear to synapse onto vasopressin-synthesizing neurons. To evaluate the functionality of these lamina terminalis ATlaR neurons, we virally delivered light-sensitive opsins and then optogenetically excited or inhibited the neurons while evaluating cardiovascular parameters or fluid intake. Optogenetic excitation robustly elevated blood pressure, water intake, and sodium intake, while optogenetic inhibition produced the opposite effects. Intriguingly, optogenetic excitation of these ATlaR neurons of the lamina terminalis also resulted in Fos induction in vasopressin neurons within the PVN and supraoptic nucleus. Further, within the PVN, selective optogenetic stimulation of afferents that arise from these lamina terminalis ATlaR neurons induced glutamate release onto magnocellular neurons and was sufficient to increase blood pressure. These cardiovascular effects were attenuated by systemic pretreatment with a vasopressin-1a-receptor antagonist. Collectively, these data indicate that excitation of lamina terminalis ATlaR neurons induces neuroendocrine and behavioral responses that increase blood pressure.

Key words: angiotensin Type 1 receptors; blood pressure; hypertension; median preoptic nucleus; renin-angiotensin system

Significance Statement

Hypertension is a widespread health problem and risk factor for cardiovascular disease. Although treatments exist, a substantial percentage of patients suffer from "drug-resistant" hypertension, a condition associated with increased activation of brain angiotensin receptors, enhanced sympathetic nervous system activity, and elevated vasopressin levels. The present study highlights a role for angiotensin Type 1a receptor expressing neurons located within the lamina terminalis in regulating endocrine and behavioral responses that are involved in maintaining cardiovascular homeostasis. More specifically, data presented here reveal functional excitatory connections between angiotensin-sensitive neurons in the lamina terminals and vasopressin neurons in the paraventricular nucleus of the hypothalamus, and further indicate that activation of this circuit raises blood pressure. These neurons may be a promising target for antihypertensive therapeutics.

Received June 14, 2020; revised Nov. 29, 2020; accepted Dec. 3, 2020

Author contributions: C.J.F., S.W.H., E.G.K., and A.D.d.K. designed research; C.J.F., S.W.H., A.R.A.,

W.S., J.A.S., and A.D.d.K. analyzed data; C.J.F., E.G.K., and A.D.d.K. wrote the paper; S.W.H., A.R.A., M.M., W.S., J.A.S., K.E., E.A.S., D.N.J., K.A.S., and A.D.d.K. performed research; S.W.H., W.S., J.A.S., K.E., and K.A.S. edited the paper.

The authors declare no competing financial interests.
This work was supported by American Heart Association Grant 17GRNT33660969 and National Institutes of Health Grants HL-125805 and HL-145028 to A.D.d.K., and HL-136595, HL-096830, and HL-122494 to E.G.K.

Correspondence should be addressed to Annette D. de Kloet at adekloet@ufl.edu.

https://doi.org/10.1523/JNEUROSCI.1600-20.2020

Copyright $\odot 2021$ the authors 


\section{Introduction}

Blood pressure and body fluid homeostasis are controlled by endocrine, autonomic, and behavioral responses that maintain blood volume and perfusion pressure at levels optimal for survival. The brain coordinates these responses, and the renin-angiotensin system (RAS) potently influences this central regulation. Along these lines, Angiotensin-II (Ang-II) acts at angiotensin type la receptors (ATlaRs) in the brain to enhance sodium and water consumption, arginine vasopressin (AVP) secretion, and sympathetic outflow (Toney and Porter, 1993; Chiodera et al., 1998; Shi et al., 2004; Zhu et al., 2005). While it is acknowledged that AT1aR (s) are localized to brain regions that are critical for these processes, and hence the maintenance of blood pressure (Rowe et al., 1990; Lenkei et al., 1997), the precise connectivity and functionality of neurons expressing ATlaR(s) have not been determined.

The endocrine and autonomic responses that maintain blood pressure and body fluid homeostasis are largely mediated by the paraventricular nucleus of the hypothalamus (PVN), which contains distinct neuronal phenotypes that control endocrine axes and sympathetic outflow (Swanson and Sawchenko, 1980). Specifically, endocrine responses are mediated by magnocellular neurons that synthesize AVP and send axons to the posterior pituitary. Excitation of these neurons elevates circulating levels of AVP, which increases blood pressure by causing vasoconstriction and renal water retention. The PVN also contains parvocellular neurons that adjust autonomic outflow via axonal projections to the rostral ventrolateral medulla (RVLM) or the spinal intermediolateral cell column. Excitation of these presympathetic neurons increases blood pressure by augmenting sympathetic outflow (Tucker and Saper, 1985; Coote et al., 1998; Cato and Toney, 2005). Stimulation of the RAS and, in particular, AT1aRs within the brain is heavily linked to AVP release and sympathetic outflow that increases blood pressure (Loń et al., 1996; Marciante et al., 2019; Pitra et al., 2019); and because the PVN expresses AT1aR, a predominating hypothesis has been that Ang-II acts directly on AVP-containing and preautonomic neurons to drive these responses. However, increasing evidence demonstrates that such neurons do not themselves express angiotensin receptors (Lenkei et al., 1995; Oldfield et al., 2001). Instead, AVP and preautonomic neurons of the PVN are engulfed by fibers and/or axon terminals originating from neurons expressing AT1aR, but perhaps, residing in other brain nuclei (de Kloet et al., 2017). In other words, it is likely that the PVN receives input from different populations of neurons that express ATlaR. In this regard, the median preoptic nucleus (MnPO), organum vasculosum of the lamina terminalis (OVLT), and subfornical organ (SFO) express ATlaRs, send projections to the PVN, and are implicated in body fluid and cardiovascular homeostasis (Stocker and Toney, 2005; Ployngam and Collister, 2007; Krause et al., 2011; Llewellyn et al., 2012).

Here, we hypothesize that neurons in the MnPO and OVLT that express AT1aR regulate body fluid homeostasis and blood pressure, and that a contributing mechanism to these effects is a direct excitatory connection between these neurons and magnocellular AVP neurons within the PVN. To test this hypothesis, studies first used neuroanatomical techniques to characterize the transmitter and projection phenotypes of neurons within the MnPO/OVLT that express AT1aR(s). Next, we used virally mediated gene transfer and Cre-loxP technology to direct the ultra-sensitive $\mathrm{Ca}^{2+}$ sensor, GCaMP6f, to the AT1aR gene (Agtrla) to examine neuronal activity in vitro during bath application of Ang-II. Subsequently, in vivo optogenetics was used to determine whether exciting or inhibiting ATlaR neurons within the MnPO/OVLT altered fluid intake, cardiovascular function, and the activity of AVP neurons. Last, we used a combination of channelrhodopsin-2 (ChR2) assisted circuit mapping and in vivo optogenetics to evaluate the functional connectivity between AT1aR neurons in the MnPO/OVLT and AVP neurons in the PVN. Collectively, these studies provide mechanistic insight into neural circuits involved in cardiovascular and fluid homeostasis and suggest that AT1aR neurons in the MnPO/OVLT exert an excitatory influence over AVP neurons that is coupled to changes in blood pressure.

\section{Materials and Methods}

Animals. All procedures were performed at the University of Florida, were approved by the Institutional Animal Care and Use Committee, and were conducted in accordance with the National Institutes of Health Guide for the care and use of laboratory animals. All mice used in these studies were male and on a C57BL/6J background, were 8-10 weeks old at the initiation of the studies, and were maintained in temperature-controlled and humidity-controlled rooms on a 12:12 h light/dark cycle with food and water available ad libitum.

Agtrla-Cre knock-in mice were generated by Biocytogen and the University of Florida and are available at The Jackson Laboratory (stock $\# 030553$ ). As described in detail in our previous publication (de Kloet et al., 2017), to express Cre-recombinase without interrupting Agtrla expression, 2A-Cre-2A-zsGreen was introduced upstream of Agtr1a three UTR via homologous recombination. Agtrla-Cre knock-in mice were used for adeno-associated virus (AAV)-mediated gene transfer and optogenetics studies. Agtrla-Cre knock in mice were also bred with stopflox-tdTomato mice (The Jackson Laboratory, stock \#007914) to verify Cre-recombinase expression throughout the MnPO and OVLT and for RNAscope ISH studies.

Viral constructs. Viral vectors used for optogenetic studies were generated by Karl Deisseroth and colleagues (Stanford University, Palo Alto, CA) and obtained from the Viral Vector Core at the University of North Carolina (Chapel Hill, NC). These AAV vectors (seroType 2) allow for the Cre-inducible expression of eYFP, the light-sensitive ion channel (ChR2), and the light-gated ion pump (halorhodopsin) and are as follows: for expression of eYFP and ChR2: AAV2-EF1a-DIOhChR2(H134R)-eYFP-WPRE (referred to as AAV-ChR2); for the expression of only eYFP: AAV2-EF1a-DIO-eYFP (referred to as AAVeYFP); and for the expression of eYFP and halorhodopsin: AAV2EF1a-DIO-eNpHR3.0-EYFP (referred to as AAV-Halo). In one study, mice were transfected with a Cre-dependent AAV (serotype 1) that allows for the expression of the GCAMP6f in ATlaR neurons localized to the MnPO/OVLT (AAV1-CAG-Flex-GCaMP6f-WPRE-SV40; obtained from Addgene). Brain slices from these mice were used for in vitro calcium imaging studies. Last, to retrogradely transfect neurons within the PVN that project to the RVLM, the AAVrg-CAG-tdTomato was used, which was obtained from Addgene (catalog \#59462) and allows for the expression of tdTomato in these preautonomic PVN neurons.

Stereotaxic surgery. Stereotaxic surgery was performed to deliver the Cre-inducible AAVs into the MnPO and OVLT of Agtrla-Cre mice. Mice were anesthetized using isoflurane, administered analgesic (buprenorphine; $0.1 \mathrm{mg} / \mathrm{kg}$ ), and prepared for stereotaxic surgery. Cre-inducible AAVs described above were injected into the MnPO/OVLT of Agtr1a-Cre mice using a picospritzer III (Parker) and the following coordinates from bregma: AP, $0.76 \mathrm{~mm}$; ML, $\pm 0 \mathrm{~mm}$; dorsoventral, -4.15 $\mathrm{mm}$. Pipettes were left in the $\mathrm{MnPO}$ for $5 \mathrm{~min}$ to allow for diffusion of the AAVs (200-300 nl) into the brain. Mice were then stereotaxically implanted with a fiber-optic post using the following coordinates from bregma: for $\mathrm{MnPO}$ (AP, $0.76 \mathrm{~mm}, \mathrm{ML} \pm 0 \mathrm{~mm}$; DV, $-3.85 \mathrm{~mm}$, from the surface of the brain) or for PVN (AP, $0.0 \mathrm{~mm}, \mathrm{ML}, 0.25 \mathrm{~mm}$; DV, $-3.85 \mathrm{~mm}$, from the surface of the brain). In one study, the AAVrgCAG-tdTomato was injected into the RVLM using the following coordinates from $\lambda$ : AP, $-1.57 \mathrm{~mm}$; ML, $\pm 1.3 \mathrm{~mm}$; DV,$-5.07 \mathrm{~mm}$, from the surface of the brain. Mice used for tracing and electrophysiological studies were not implanted with fiber-optic posts. All AAV-injected mice were 
allowed to recover for a minimum of 2 weeks before beginning subsequent procedures.

Assessment of blood pressure in response to optogenetic manipulations. To test whether ATlaR-expressing neurons within the $\mathrm{MnPO} /$ OVLT regulate blood pressure, in vivo optogenetic experiments were conducted in anesthetized animals. Agtrla-Cre mice were injected with Cre-inducible AAVs (AAV-ChR2, AAV-Halo, or AAV-eYFP [control]) into the MnPO and implanted with fiber-optic posts as described above. Six weeks later, optogenetic experiments evaluated acute blood pressure responses to optogenetic stimulation of ATlaR-expressing neurons within the MnPO/OVLT or ATlaR-expressing fibers within the PVN.

Catheterization and blood pressure recordings were done under isoflurane anesthesia. Although isoflurane does reduce baseline blood pressure and heart rate relative to levels that are observed in awake mice, the concentrations that are used here ( $\sim 1 \%-1.5 \%)$ allow for stable blood pressure and heart rate measurements during prolonged recordings ( $\sim 30-240 \mathrm{~min}$ ) (Janssen et al., 2004; Szczesny et al., 2004; Constantinides et al., 2011a,b). Blood pressure was measured using a Millar catheter (model SPR1000, Millar) connected to a PowerLab signal transduction unit (AD Instruments) and was recorded and analyzed using Labchart8 software (AD Instruments). An incision $(1 \mathrm{~cm})$ was made on the midline of the ventral neck to expose the left carotid artery. Subsequently, a 1-cm-long segment of the carotid artery was separated from the vagus nerve using two sutures (size: 6-0; silk); the cranial end of the segment was permanently ligated and the caudal end of the segment was temporarily occluded. Next, the carotid artery was punctured and the catheter was slid into the artery lumen and then secured using suture. Suture was then used to close the skin during the experimental protocol.

The first cardiovascular experiment examined the impact of optogenetically exciting the soma of these AT1aR-expressing neurons in the MnPO/OVLT on cardiovascular parameters using ChR2. For this experiment, fiber-optic posts were positioned directly above the MnPO. After a period of baseline recording of cardiovascular parameters, AAV-ChR2 mice and littermate AAV-eYFP controls were stimulated with laser-generated blue light $(473 \mathrm{~nm}$; Shanghai Laser and Optics) for $10 \mathrm{~min}(20 \mathrm{~ms}$ pulses; $15 \mathrm{~Hz} ; 60 \mathrm{~s}$ on/off). Cardiovascular parameters were recorded for a period of $50 \mathrm{~min}$ after the onset of blue light stimulation. In order to inhibit this same population of neurons, AAV-Halo mice and their littermate AAVeYFP controls similarly underwent a period of baseline recording. Then, these mice were subjected to 10 min of laser-generated yellow light (561 nm; 10mW; Shanghai Laser and Optics), while cardiovascular parameters were recorded.

In the final study, AAV-ChR2 mice and littermate AAV-eYFP controls were subjected to blue light stimulation of nerve terminals that arise from these ATlaR-containing neurons of the MnPO and OVLT and are localized to the PVN. These recordings were performed before and after the application of a vasopressin 1a receptor (V1aR) antagonist (SR 49059 , Tocris Bioscience; $2 \mathrm{mg} / \mathrm{kg}$, i.p.) or saline vehicle. The protocol is as follows. Fiber-optic posts were positioned over the PVN of AAVChR2 or AAV-eYFP mice as described above. After a period of stable baseline blood pressure recording, mice underwent a $60 \mathrm{~s}$ period of blue light stimulation $(20 \mathrm{~ms}$ pulses; $15 \mathrm{~Hz})$. Mice were left undisturbed for 10-12 min until all the recording signals return to their baseline control values. Afterwards, mice were administered VlaR antagonist $(2 \mathrm{mg} / \mathrm{kg}$, IP) or saline vehicle. Both systolic blood pressure (SBP) and mean arterial pressure baselines were significantly reduced in $<15 \mathrm{~min}$ by the $\mathrm{V} 1 \mathrm{aR}$ antagonist. Once blood pressures were again stable, mice were subjected to blue light stimulation for $60 \mathrm{~s}(20 \mathrm{~ms}$ pulses; $15 \mathrm{~Hz})$.

Assessment of ad libitum drinking behavior in response to optogenetic manipulations. To test whether AT1aR-expressing neurons within the $\mathrm{MnPO}$ regulate water and sodium intake, we conducted in vivo optogenetic experiments in freely moving mice. Mice were administered the AAVs and implanted with fiber-optic posts as described above. After recovery, mice were handled for 1 week and then habituated to the intake chamber for 1 week. The intake chamber consists of four separate lanes, each of which has one lixit containing water and another containing $0.3 \mathrm{M} \mathrm{NaCl}$ exposed at the far end. The lixits are blocked off by a gate to allow for acclimation before the gate's removal. During habituation, mice were tethered and given access to the lixits for $10 \mathrm{~min}$ for $4 \mathrm{~d}, 2 \mathrm{~d}$ of which the mice had been water deprived overnight to promote drinking.

On test days, during the light phase between 8:00 A.M. and 12:00 P. M., the mice were tethered to the light sources, placed into the intake chamber, and allowed $3 \mathrm{~min}$ of acclimation. The light sources were then initiated and the gate was lifted simultaneously. For mice that received AAV-ChR2 and AAV-eYFP, the blue light stimulation parameters were as follows and were sustained for a period of $10 \mathrm{~min}$ : $20 \mathrm{~ms}$ pulses, $15 \mathrm{~Hz}, 60 \mathrm{~s}$ on followed by $60 \mathrm{~s}$ off. For mice that received AAV-Halo, the yellow-light stimulation was continuous and sustained for a period of $10 \mathrm{~min}$. Licks were recorded and analyzed using the Noldus Information Technology's EthoVision XT 13.0. All mice were randomized to receive either the Light on condition or Light off condition on Test day 1 , and the opposite condition was delivered on Test day 2 . These conditions were repeated for a total of $4 \mathrm{~d}$.

Assessment of deprived drinking behavior in response to optogenetic manipulations. Two additional test days followed the same parameters as described above, but in these studies the animals were water-deprived overnight before test day stimulation. Also, for these studies, mice given the AAV-eYFP control virus were stimulated with the yellow light. All mice were randomized to receive either the Light on condition or Light off condition on test day 1 , and the opposite condition was delivered on test day 2. Deprived licks were recorded and analyzed (Noldus).

Assessment of Fos immunoreactivity in response to optogenetic stimulation. Mice used for the drinking experiments were also used to evaluate Fos immunoreactivity in response to optogenetic stimulation. After allowing the mice to recover for 1 week from these previous studies, mice underwent a $5 \mathrm{~d}$ period of habituation to the tethering procedure in their home cages. During this habituation period, mice were brought into the procedure room, tethered to the laser light source, and then allowed to freely move in their home cages for $60 \mathrm{~min}$. On the day of the test, mice were brought into the procedure room, tethered, and allowed to recover from the tethering procedure for $90 \mathrm{~min}$. At this time, AAVChR2 and AAV-eYFP mice underwent the same optical stimulation protocol that is described above ( $10 \mathrm{~min} ; 10 \mathrm{~mW}, 15 \mathrm{~Hz} ; 60 \mathrm{~s}$ on/off). Ninety minutes later, mice were killed, perfused, and tissue was collected as described below.

Tissue collection and sectioning. To evaluate virus and fiber-optic post placement and also to evaluate Fos immunoreactivity subsequent to a $10 \mathrm{~min}$ period of optogenetic stimulation, mice were anesthetized with pentobarbital and perfused transcardially with $0.15 \mathrm{M} \mathrm{NaCl}$ followed by $4 \%$ PFA. Brains were then postfixed for 3-4 h, after which they were stored in $30 \%$ sucrose until they were sectioned using a CM3050 S cryostat (Leica Microsystems). For ISH experiments, perfused mouse brains were sectioned at $20 \mu \mathrm{m}$ into six serial sections and immediately mounted onto SuperFrost Plus Gold microscope slides. After air drying at room temperature for 20-30 min, slides were stored at $-80^{\circ} \mathrm{C}$ until further processing. All solutions were prepared with DEPC-treated water and filtered using a $0.22 \mu \mathrm{m}$ filter. Tissue collection and sectioning were performed in RNase-free conditions. For IHC studies, mouse brains were sectioned at $30 \mu \mathrm{m}$ into four serial sections and stored in cryoprotectant solution at $-20^{\circ} \mathrm{C}$ until further processing.

Immunohistochemistry. Immunohistochemistry was performed on free-floating tissue sections using protocols that we have described previously (de Kloet et al., 2017). In brief, brain sections were removed from cryoprotectant solution and rinsed 5 times for $5 \mathrm{~min}$ each in $50 \mathrm{~mm}$ potassium PBS. Sections were then incubated in blocking solution ( $2 \%$ normal donkey serum and $0.2 \%$ Triton X-100 in $50 \mathrm{~mm}$ potassium PBS) for $2 \mathrm{~h}$ at $22^{\circ} \mathrm{C}-25^{\circ} \mathrm{C}$ and then in the primary antibody (diluted in blocking solution) for $18-24 \mathrm{~h}$ at $4^{\circ} \mathrm{C}$. Sections were again rinsed 5 times for $5 \mathrm{~min}$ each before incubation in the secondary antibody (also diluted in blocking solution) for $2 \mathrm{~h}$ at $22^{\circ} \mathrm{C}-25^{\circ} \mathrm{C}$. After a final series of rinses, sections were mounted onto slides, allowed to air dry, and then coverslipped using polyvinyl alcohol mounting medium. All primary antibodies were characterized by the manufacturers and in previously published studies (Ben-Barak et al., 1985; Jessberger et al., 2008; Kádár et al., 2010). The antibodies and dilutions used are as follows: GFP (Invitrogen, A10262; 1:1000), vasopressin-neurophysin (AVP; H. Gainer, National Institutes 

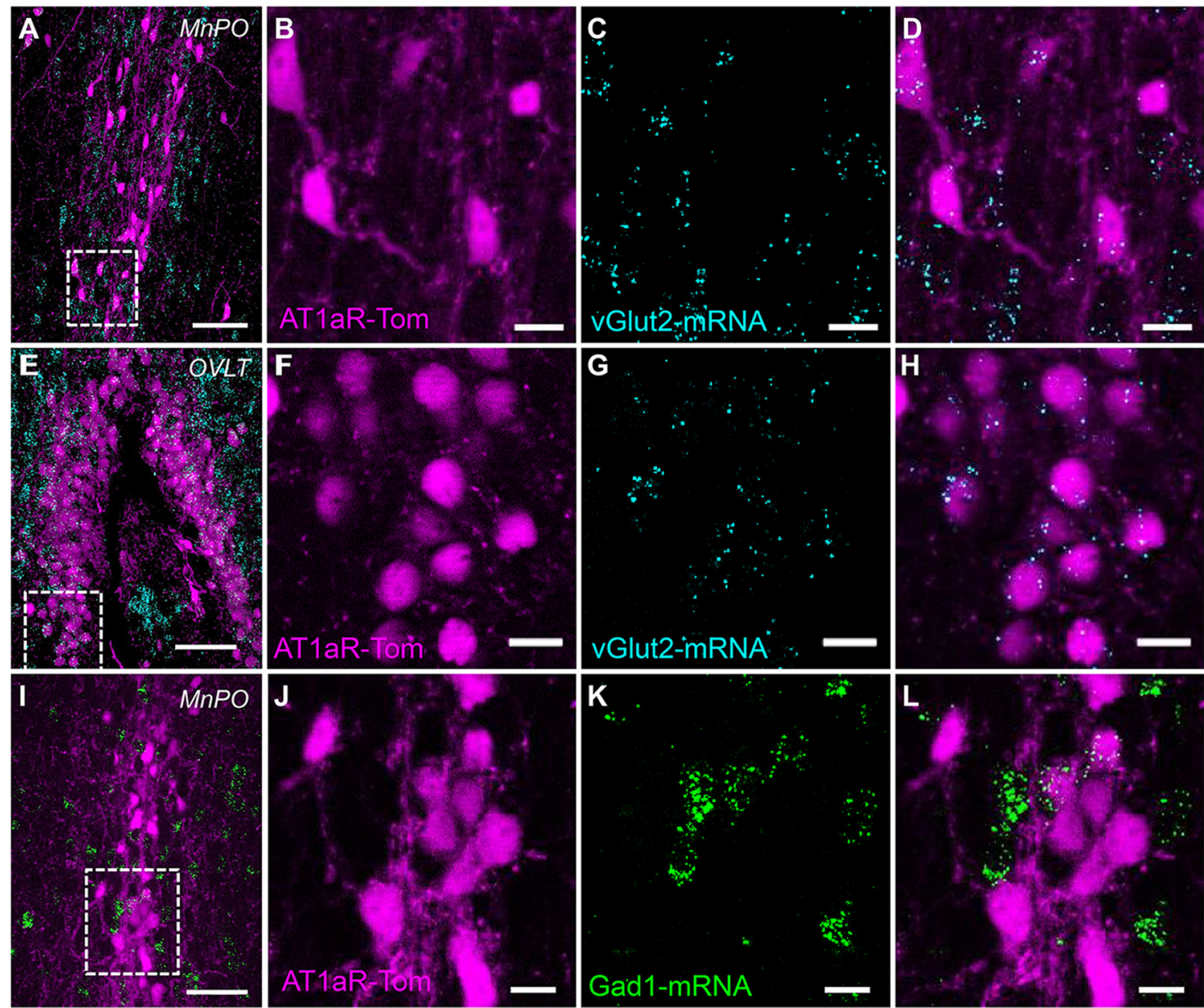

Figure 1. AT1aR neurons within the MnP0 and OVLT are predominantly glutamatergic. Images of the $(\boldsymbol{A}-\boldsymbol{D}) \mathrm{MnPO}$ and $(\boldsymbol{E}-\boldsymbol{H})$ OVLT collected from an Agtr1a-tdTomato (AT1aR-Tom) reporter mouse depicting $(\boldsymbol{B}, \boldsymbol{F}) v G$ lut2 mRNA in cyan, $(\boldsymbol{C}, \boldsymbol{G})$ AT1aR-tdTom in magenta, and $(\boldsymbol{A}, \boldsymbol{D}, \boldsymbol{E}, \boldsymbol{H})$ the merged images. $\boldsymbol{I}-\boldsymbol{L}$, Images of the MnP0 collected from an AT1aR-Tom reporter mouse depicting (J) Gad1 mRNA in green, (K) AT1aR-Tom in magenta, and ( $\boldsymbol{L}$ ) the merged image. $n=4$ mice. Scale bars: $\boldsymbol{A}, \boldsymbol{E}, \boldsymbol{I}, 50 \mu \mathrm{m} ; \boldsymbol{B}-\boldsymbol{D}, \boldsymbol{F}-\boldsymbol{H}, \boldsymbol{J}-\boldsymbol{L}, 20 \mu \mathrm{m}$.

of Health; PS-4; 1:400), and Fos (Santa Cruz Biotechnology, sc52; 1:1000). Secondary antibodies (Jackson ImmunoResearch Laboratories) were raised in donkey and used at a 1:500 dilution.

RNAscope ISH. RNAscope ISH was performed as per the manufacturer's instructions using the RNAscope Multiplex Fluorescent Reagent Kit (Advanced Cell Diagnostics) with slight modifications to the pretreatment procedure that were previously described (de Kloet et al., 2016). The probes used for these assays were as follows: $v$ Glut2 (Mm-Slc17a6-C3; catalog \#319171-C3); Gad1 (Mm-Gad1-C3; catalog \#400951-C3); DapB (Negative control probe-DapB-C3; catalog \#310043-C3); and Ubc (MmUbc-C3; catalog \#310771-C3). The DapB and Ubc served as negative and positive control probes, respectively.

Image capture and processing. Images were captured and processed using Axiovision 4.8.2 software and a Carl Zeiss AxioImager fluorescent Apotome microscope. For dual IHC/RNAscope ISH depicted in Figure $1, z$ stacks of the proteins and transcripts of interest were captured at $20 \times$ magnification throughout the ROIs using neuroanatomical landmarks. In all cases, $z$ steps were set at $0.5 \mu \mathrm{m}$, with an average of 20 optical sections per image. These $z$ stacks were used to generate the projection images depicted throughout the manuscript. For each experiment, sections hybridized with the positive control probes were used to determine the exposure time and image processing required to provide optimal visualization of mRNA signal. As described in detail (de Kloet et al., 2016), these same parameters were then used for visualization of mRNA transcripts of interest to assess background fluorescence in sections hybridized with negative control probe $(D a p B)$. Importantly, using these exposure times and image-processing parameters there was minimal or no fluorescence in sections hybridized with the negative control probe. For IHC studies depicted in Figures 3 and 5, images were captured at $5 \times, 10 \times$, or $20 \times$ magnifications. Finally, to conduct the survey of projection sites of ATlaR MnPO/OVLT neurons that is highlighted in Table 1, coronal sections through the ROIs were scanned using a Nikon $\mathrm{C} 2+$ confocal microscope at $20 \times$ and high-resolution large images were generated. All final figures were then prepared using Adobe Photoshop where the brightness and contrast was adjusted to provide optimal visualization.

Image analysis. The $20 \times$ magnification $z$ stacks were used for the manual determination of the percentage of ATlaR-tdTomato neurons that contain Gad1- or vGlut2. AT1aR-tdTomato were considered to contain the RNA if at least three visible transcripts, defined as an individual punctate dot, were observed within the volume of tdTomato fluorescence. To probe for the presence of putative projection sites of $\mathrm{MnPO} /$ OVLT neurons that express ATlaR, coronal sections of AAV-eYFP mice were scored as containing dense, moderate, or sparse fibers. ROIs are listed in Table 1. To evaluate the presence of likely appositions of AT1aR MnPO/OVLT neuronal axons onto AVP neurons, individual $z$ stacks $(40 \times$ and $20 \times)$ were evaluated to assess whether the fibers terminate on the surface of the AVP-labeled cells. These qualitative assessments of apparent contacts onto magnocellular, as well as parvocellular, neurons within the PVN were then evaluated using in vitro ChR2 assisted circuit mapping and patch-clamp electrophysiology as described below.

Acute brain slice preparation. Mice were anesthetized using a single intraperitoneal injection of ketamine $(80-100 \mathrm{mg} / \mathrm{kg})$ and then decapitated with a small animal guillotine. Brains were rapidly extracted, and $300 \mu \mathrm{m}$ coronal sections were prepared with a Leica Microsystems VT$1000 S$ vibratome. ACSF used during dissection and sectioning was icecold and contained the following (in $\mathrm{mm}$ ): 205 sucrose, 10 dextrose, 
Table 1. Qualitative summary of the density of fibers arising from AT1aR neurons of the MnPO/OVLT ${ }^{\mathrm{a}}$

\begin{tabular}{ll}
\hline & \multicolumn{2}{l}{ Fiber density } \\
\cline { 2 - 2 } ROI & None Sparse Moderate Dense \\
\hline
\end{tabular}

Dorsolateral preoptic nucleus

BNST (ventral medial)

BNST (ventral lateral)

BNST (dorsal medial)

PVN (posterior)

PVN (parvocellular)

PVN (magnocellular)

Periventricular hypothalamic nucleus

SFO

SON

Arcuate nucleus

DMH (ventral)

DMH (compact)

DMH (dorsal)

Perifornical area

Lateral hypothalamus

Median eminence

Ventrolateral periaqueductal gray

Dorsal and dorsolateral periaqueductal gray

Dorsal raphe/median raphe

Locus coeruleus

Lateral parabrachial nucleus region

Raphe pallidus

Commissural nucleus of the solitary tract/central canal $x$

${ }^{a}$ This analysis was performed on brains collected from Agtr1a-Cre mice that received the AAV-eYFP into the MnPO/OVLT.

$1 \mathrm{MgSO}_{4}, 2 \mathrm{KCl}, 1.25 \mathrm{NaH}_{2} \mathrm{PO}_{4}, 1 \mathrm{CaCl}_{2}$, and $25 \mathrm{NaHCO}_{3}$. Sections were then transferred for $30 \mathrm{~min}$ to a low-calcium ACSF maintained at $37^{\circ}$ C that contained the following (in mM): $124 \mathrm{NaCl}, 10$ dextrose, $3 \mathrm{MgSO}_{4}$, $2.5 \mathrm{KCl}, 1.23 \mathrm{NaH}_{2} \mathrm{PO}_{4}, 1 \mathrm{CaCl}_{2}, 25 \mathrm{NaHCO}_{3}$. After a 30 min incubation period at $37^{\circ} \mathrm{C}$, slices were allowed to passively equilibrate to room temperature in the same ACSF. In vitro experiments were conducted at $30^{\circ} \mathrm{C}$ in ACSF that contained the following (in $\mathrm{mm}$ ): $126 \mathrm{NaCl}, 11$ dextrose, $1.5 \mathrm{MgSO}_{4}, 3 \mathrm{KCl}, 1.2 \mathrm{NaH}_{2} \mathrm{PO}_{4}, 2.4 \mathrm{CaCl}_{2}$, and $25 \mathrm{NaHCO}_{3}$. All three types of ACSF described above were continuously saturated with $95 \%$ oxygen and $5 \%$ carbon dioxide, and had a $\mathrm{pH}$ of $\sim 7.3$.

Whole-cell recording. Patch pipettes were pulled using a P97 Flaming/Brown Pipette Puller (Sutter instrument) from $1.5 \mathrm{~mm} / 0.86$ $\mathrm{mm}$ borosilicate glass (BF150-86-10, Sutter instrument). Open tip resistance was 4-6 $\mathrm{M} \Omega$ when filled with an internal solution that contained the following (in $\mathrm{mM}$ ): $125 \mathrm{~K}$-gluconate, 10 phosphocreatine, $1 \mathrm{MgCl}_{2}, 10$ HEPES, 0.1 EGTA, $2 \mathrm{Na}_{2}$-ATP, and $0.25 \mathrm{Na}_{3}$-GTP. This solution was $\mathrm{pH}$-adjusted to 7.3 using $\mathrm{KOH}$ and was volume-adjusted to $\sim 295$ mOsm. Whole-cell patch-clamp data were acquired using a MultiClamp 700B amplifier paired with a Digidata 1440A digitizer (Molecular Devices) and pCLAMP 10 software. Real-time analysis of electrophysiological data was performed with custom software written for this project using Python 3.7 and the pyABF module. Offline analysis of all electrophysiological data was performed using custom software written in OriginC (OriginLab) by C.J.F. For experiments involving in vitro optogenetics, a TTL-controlled LED light source (X-Cite 110LED) was configured to deliver light pulses through a $40 \times$ water-immersion lens. Wavelength was controlled by passing light through filter cubes (Omega Optical) with blue (XF404) or green (XF414) emission filters.

In vitro GCaMP6f imaging. Experiments involving GCaMP6f imaging used brain slice preparation techniques and extracellular solutions identical to that for patch-clamp electrophysiology experiments. An epifluorescence light source (X-Cite 110LED), in combination with a blue/ green filter set (XF404, Omega Optical), was used with a low-power $(4 \times)$ objective lens. Ionotropic glutamate receptor antagonists $(20 \mu \mathrm{M}$ DNQX and $40 \mu \mathrm{M}$ AP5) and GABA receptor antagonists (100 $\mu \mathrm{M}$ PTX and $10 \mu \mathrm{M}$ CGP 55845) were continuously applied to reduce basal synaptic transmission. Micro-manager software (Edelstein et al., 2010) was configured to power-on the epifluorescence light source and acquire an image once every $20 \mathrm{~s}$. Fiji software (Schindelin et al., 2012) was used to measure GCaMP6f fluorescence intensity within a manually defined ROI encompassing the MnPO/OVLT. In order to correct for slow effects of photobleaching over the course of the experiment, fluorescence intensity in each ROI was divided, in each frame, by fluorescence intensity of an adjacent ROI that had no GCaMP6f-positive neurons. After that operation, percent change in fluorescence intensity $(\Delta \mathrm{F} / \mathrm{F})$ was calculated as $\left(\mathrm{F}-\mathrm{F}_{0}\right) / \mathrm{F}_{0}$, where $\mathrm{F}$ is mean fluorescence intensity in the ROI, and $\mathrm{F}_{0}$ is the mean fluorescence intensity observed in the same ROI over a $3 \mathrm{~min}$ baseline period before drug application. The response to Ang-II is then reported as the mean $\Delta \mathrm{F} / \mathrm{F}$ observed over a $5 \mathrm{~min}$ period beginning with the application of Ang-II. In a separate series of experiments, procedures were performed exactly as above, except that losartan $(10 \mu \mathrm{M})$ was continuously applied along with the glutamate and GABA receptor antagonists.

Biocytin labeling and post hoc immunohistochemistry. In experiments where post hoc immunohistochemical assessment of electrophysiologically characterized neurons was used, pipette solutions supplemented with biocytin (5 mM) were used. Upon completion of each experiment, the patch pipette was withdrawn diagonally under visual control. Slices where the recorded cell remained intact and stationary as the pipette was withdrawn were saved for immunohistochemical analysis and transferred to $10 \%$ formalin and stored at $4^{\circ} \mathrm{C}$ for at least $12 \mathrm{~h}$. Slices $(300 \mu \mathrm{m}$ thick) were then transferred to 12 -well plates where each slice was processed individually using a $1 \mathrm{ml}$ reaction volume. Slices were permeabilized for $72 \mathrm{~h}$ in blocking solution (2\% BSA, $2 \%$ normal goat serum, $0.1 \%$ sodium azide, and $0.1 \%$ Triton X-100 in PBS). Slices were then exposed to mouse anti-vasopressin-neurophysin (H. Gainer, National Institutes of Health; PS-4; 1:100) and rabbit anti-GFP (Abcam AB6556; 1:1000) antibodies in blocking solution for $48 \mathrm{~h}$. Following 5 times for 5 min PBS washes, goat anti-rabbit 488 (Thermo Fisher Scientific, A27034; 1:500) and goat antimouse 594 (Thermo Fisher Scientific, A11005; 1:500) antibodies were applied in combination with 647-conjugated streptavidin (Thermo Fisher Scientific, S32357; 1:2000) in blocking solution for $24 \mathrm{~h}$. Following 5 times for 5 min PBS washes, slices were transferred to glass slides and immediately coverslipped with an aqueous mounting medium (VECTASHIELD, Vector Laboratories H1000) and permitted to rest for $24 \mathrm{~h}$ before imaging. Imaging was performed using an Olympus BX51WI upright microscope and single photon excitation (Excelitas X-Cide 110LED illumination, XF404, XF406, or XF407 filter cube, QImaging QICAM Fast 1394 camera, and National Institutes of Health ImageJ software) or two-photon excitation (Coherent Mira Optima 900-F mode-locked titanium: sapphire laser tuned to $810 \mathrm{~nm}$, Hamamatsu R3896 SEL photomultiplier tube, and Prairie Technologies PrairieView software) through a $20 \times$ air objective lens.

Experimental design and statistics. As highlighted in Animals, studies were conducted in adult male mice on a C57BL/6J background. Appropriate sample sizes were predetermined by way of power analyses that were performed using the software G*power, and expected variances and effect sizes that were based on our preliminary and published studies (de Kloet et al., 2017). In all cases, group sizes are indicated in the figure legends or Results, and $p<0.05$ was considered statistically significant. Additionally, for these experiments, comparisons are made between the experimental groups (AAV-ChR2 or AAV-Halo) and controls delivered the AAV-eYFP during Light-on and Light-off conditions.

Cardiovascular parameters (i.e., SBP and heart rate) are reported as change from the baseline (Light-off) condition for each mouse, and statistical significance was ascertained via two-way ANOVA (time and AAV condition as within- and between-subjects factors, respectively) and subsequent Bonferroni's post hoc analyses. Drinking behavior was analyzed by determining a total number of licks for the Light on versus Light off condition and performing a two-way ANOVA with light and AAV conditions as the within- and between-subjects factors, respectively. Analysis of total Fos-positive nuclei and the percentage of AVP neurons that contain Fos-positive nuclei within the ROIs were similarly analyzed using a two-way ANOVA and Bonferroni's post hoc analyses, with brain region and AAV condition as the within- and between- 

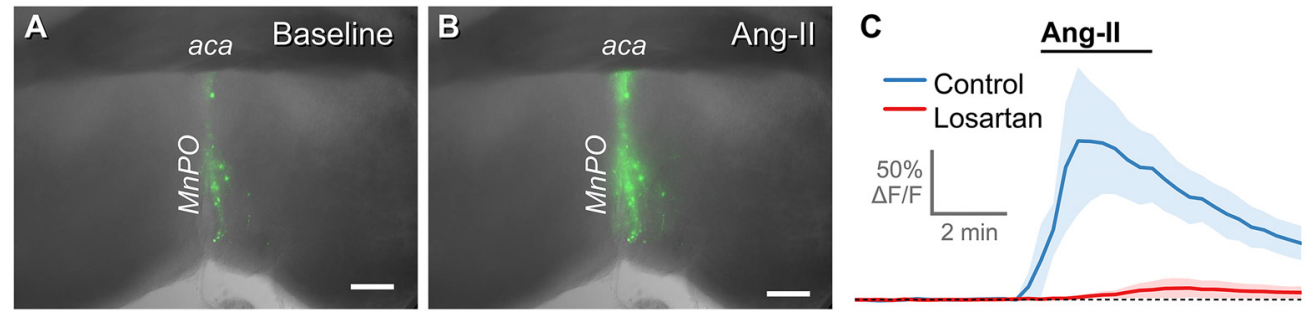

Figure 2. Angiotensin-II excites neurons in the MnP0/OVLT, and this response is attenuated by antagonism of AT1aR. $A$, AAV delivery of Cre-dependent GCaMP6f into the MnP0/OVLT of Agtr1a-Cre mice mouse selectively transforms AT1aR neurons. B, Exposure to Ang-II (1 $\mu \mathrm{m}, 3 \mathrm{~min}$ ) increases fluorescence in GGaMP6f-expressing neurons. C, The excitatory action of Ang-II ( $n=6$, blue trace) is attenuated by pretreatment with Losartan (10 $\mu \mathrm{m}, n=4$, red trace). aca, anterior commissure. Scale bars, $250 \mu \mathrm{m}$.

subjects factors, respectively. Specific details of the experimental design are highlighted above in the following sections: Assessment of blood pressure in response to optogenetic manipulations; Assessment of ad libitum drinking behavior in response to optogenetic manipulations; Assessment of deprived drinking behavior in response to optogenetic manipulations; and Assessment of Fos immunoreactivity in response to optogenetic stimulation. Finally, differences in $\Delta \mathrm{F} / \mathrm{F}$ reported by GCaMP6f across groups were evaluated using an unpaired Student's two-sample $t$ test (null hypothesis: Group 1 mean $=$ Group 2 mean), while the mean change in evoked response amplitude observed in individual cells in response to bath-applied glutamate receptor antagonists was evaluated using a ratio paired $t$ test (null hypothesis: mean of the $\log 10$ values of all paired ratios, treated/baseline, equals 0 ; see Fig. 6D).

\section{Results}

$\mathrm{AT} 1 \mathrm{aR}(\mathrm{s})$ are predominantly expressed on glutamatergic neurons of the MnPO/OVLT

Initial studies evaluated the transmitter phenotype of ATlaR neurons of the MnPO and OVLT. In order to accomplish this, the Cre-loxP system was used to generate AT1aR-tdTomato reporter mice. We have previously validated the utility of these ATlaR-tdTomato reporter mice by verifying that the majority ( $\sim 97 \%)$ of AT1aR-tdTomato cells express AT1aR mRNAs (Agtr1a; de Kloet et al., 2017). In order to determine the percentage of AT1aR-tdTomato cells within the MnPO and OVLT that are glutamatergic, RNAscope ISH for vGlut2 was performed on brain sections. Figure $1 A-D$ depicts the extent of colocalization between AT1aR-tdTomato and vGlut2, in the $\mathrm{MnPO}$, while Figure $1 E-H$ illustrates the extent of colocalization in the OVLT. Quantification of these ISH images revealed that $78 \%$ of AT1aR-tdTomato cells within these areas express vGlut2 (776 of 992 AT1aR-tdTom cells; $n=4$ mice). Importantly, these images also highlight that not all vGlut2 mRNA is localized to ATlaR-tdTomato cells, suggesting that ATlaR is likely not expressed in all glutamatergic neurons within these areas.

Using the same approach, we further found that $\sim 22 \%$ of ATlaR-tdTomato neurons in the MnPO/OVLT express Gad1 (222 of 1016 ATlaR-tdTom cells; $n=4$ mice; Fig. $1 I-L)$. Gad1 is often used as a marker for GABAergic neurons (Tamamaki et al., 2003); however, more recent evidence indicates that many neurons in the preoptic area that express Gad1 also express Vglut2 and not Vgat, so are presumably glutamatergic, not GABAergic (Moffitt et al., 2018). Thus, it is possible that some or all of the Gad1 cells may have represented glutamatergic neurons, not GABAergic ones.

AT1aR neurons of the MnPO/OVLT are activated by Ang-II In vitro calcium imaging was used to determine the impact of the exogenous application of Ang-II on the activity of the AT1aR neurons of the MnPO/OVLT and to assess the utility of AT1aR-
Cre knock-in mice for targeting neurons that contain functional ATlaR(s). Agtrla-Cre mice were injected with Cre-inducible AAV-GCaMP6f in the MnPO/OVLT. Three weeks later, coronal brain slices were made through the MnPO/OVLT. Ang-II ( $1 \mu \mathrm{M})$ was then bath-applied for $3 \mathrm{~min}$ while continuously monitoring the fluorescence of AT1aR-expressing neurons. Bath application of Ang-II caused AT1aR-expressing neurons within the MnPO/ OVLT to rapidly and transiently increase GCaMP6f fluorescence, consistent with increased activity. Figure $2 A, B$ illustrates GCaMP6f fluorescence before and during bath application of Ang-II in a representative slice. Summary data (Fig. 2C) indicate that pretreatment with the selective AT1aR antagonist losartan $(10 \mu \mathrm{M})$ significantly attenuated the response to Ang-II $(45.8 \pm 7.8 \%$ vs $3.3 \pm 2.5 \% \Delta \mathrm{F} / \mathrm{F}$ observed in control conditions vs presence of losartan, respectively, $n=6,4, p=0.0028)$. These results demonstrate that Ang-II excites neurons in the $\mathrm{MnPO} /$ OVLT that express AT1aR(s) and provides functional evidence that Cre-recombination faithfully tracks Agtrla gene expression in our mouse model.

\section{Mapping of AT1aR projections from the MnPO/OVLT to other forebrain nuclei}

The next objective was to determine the projection sites of MnPO/OVLT neurons that express ATlaR. To accomplish this, we conducted an anterograde neuronal tract tracing study, in which a Cre-dependent AAV that expresses eYFP was delivered into the MnPO/OVLT of Agtr1a-Cre mice (Fig. 3A). This leads to expression of eYFP only in AT1aR-Cre cells of the MnPO/ OVLT. Analysis of fibers/terminals was focused primarily on key cardioregulatory areas that are known to receive neural input from the MnPO/OVLT (Table 1). Figure $3 B$ demonstrates expression of eYFP in AT1aR MnPO/OVLT neurons. Figure 3C, $D$ depicts fibers arising from these ATlaR MnPO/OVLT neurons within the SFO and bed nucleus of the stria terminalis (BNST), two areas known to receive neural input from the MnPO/OVLT. As highlighted in Table 1, dense fibers were also observed in other hypothalamic brain regions (e.g., PVN, supraoptic nucleus [SON], and dorsomedial hypothalamus [DMH]). Furthermore, sparse to moderate levels of fibers were in some midbrain/hindbrain areas (e.g., portions of the periaqueductal gray), but none was noted in the commissural nucleus of the solitary tract.

Dual-label immunohistochemistry performed on brains sections from 8 mice revealed that, within the PVN, projections originating from ATlaR-expressing neurons in the $\mathrm{MnPO} /$ OVLT come into close proximity with neurons that synthesize AVP (Fig. 3E,F), suggestive of connectivity that influences the systemic release of AVP. 
A

(i)

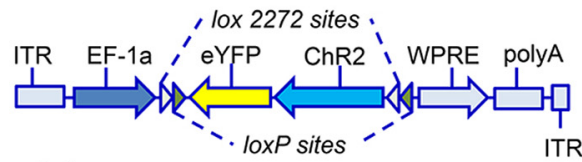
$\sqrt{ }$

(ii)

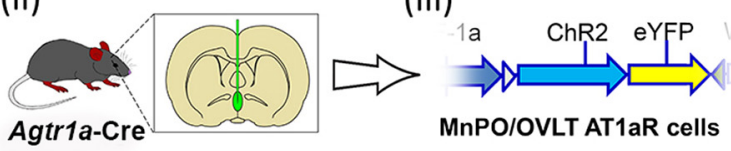

B
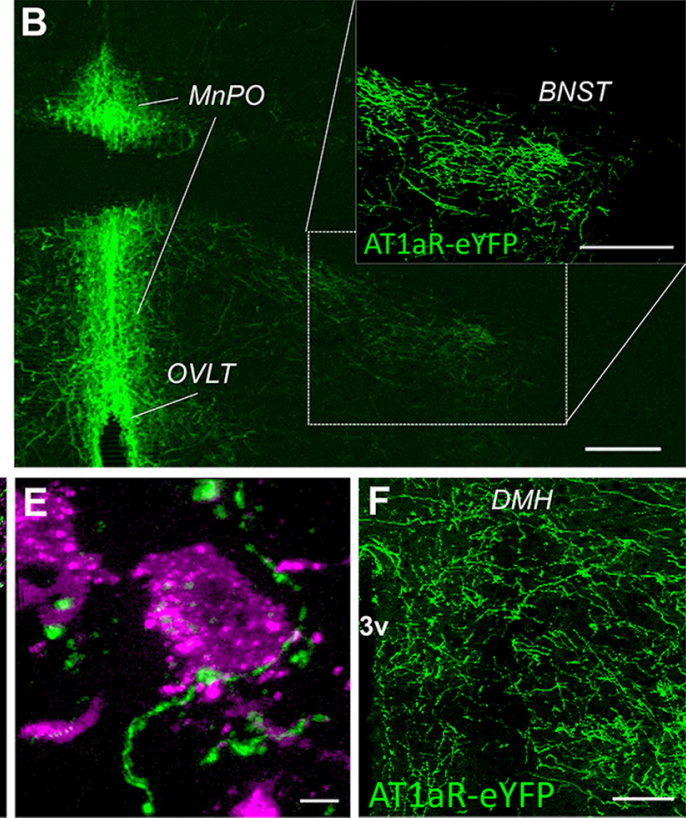

Figure 3. AT1aR neurons of the MnP0/OVLT send projections to brain regions involved in body fluid homeostasis and blood pressure regulation. $\boldsymbol{A}$, Schematic illustrating (Ai) the viral construct used to direct the Cre-dependent expression of ChR2 and eYFP to neurons within the MnPO/OVLT of the Agtr1a-Cre mouse; (Aii) the injection of the AAV into the MnP0/OVLT; and (Aiii) the subsequent Cre-mediated inversion of the dual floxed eYFP and ChR2 into the correct orientation. $\boldsymbol{B}$, Coronal section through the OVLT and MnPO depicting Cre-dependent expression of eYFP (green) in the somas of neurons expressing AT1aR and their fibers in the BNST (dashed box). Coronal section through the (C) SF0 demonstrating eYFP-labeled axons originating from AT1aR neurons of the MnP0/OVLT. D, E, Unilateral coronal section through the PVN illustrating that axons (green) arising from neurons in the MnP0/OVLT that express AT1aR make appositions onto AVP (magenta) synthesizing neurons. Coronal section through the (F) DMH demonstrating eYFP-labeled axons originating from AT1aR neurons of the MnP0/OVLT. 3v, Third cerebral ventricle. Images are representative of 8 mice. Scale bars: $\boldsymbol{B}, 200 \mu \mathrm{m} ; \boldsymbol{C}, \boldsymbol{D}, 50 \mu \mathrm{m} ; \boldsymbol{E}, 5 \mu \mathrm{m} ; \boldsymbol{F}, 100 \mu \mathrm{m}$.

\section{Optogenetic stimulation of AT1aR-containing neurons of} the MnPO/OVLT potently influences SBP and fluid intake Prior studies have revealed important roles for the $\mathrm{MnPO}$ and OVLT in the autonomic, endocrine, and behavioral responses that maintain body fluid homeostasis (e.g., Buggy et al., 1975; Buggy and Fisher, 1976; Faulk et al., 2017a,b). Therefore, we hypothesized that the activity of AT1aR neurons within these brain nuclei is coupled to changes in blood pressure and drinking behavior. To test this hypothesis, Agtrla-Cre mice were delivered a Cre-dependent AAV to direct the expression of the light-sensitive cation channel ChR2 and the eYFP fluorophore to AT1aR neurons residing in the MnPO/OVLT and then implanted with fiber-optic posts immediately dorsal to the same area. Control mice received a Cre-dependent AAV that only expresses the eYFP fluorophore. In vitro whole-cell patch-clamp recordings demonstrated that blue light pulses $(15 \mathrm{~Hz})$ reliably caused ATlaR neurons in the MnPO/OVLT to generate action potentials (Fig. $4 A-C$ ). Consequently, we used these optogenetic stimulation parameters to evaluate how in vivo excitation of $\mathrm{AT} 1 \mathrm{aR}$ neurons affects blood pressure, heart rate, and fluid intake. Three weeks after viral delivery, mice were anesthetized, a Millar catheter was implanted into the carotid artery, and the fiber-optic implant, capable of delivering light pulses to the $\mathrm{MnPO} / \mathrm{OVLT}$, was connected to a laser light source. As highlighted in Figure $4 D$, blue light stimulation significantly elevated SBP in mice that were injected with AAV-ChR2 but had no effect in mice that received the control virus expressing only the eYFP fluorophore (AAV-eYFP significant interaction; $F_{(55,385)}=2.1$; $p<0.0001)$. Interestingly, these elevations in SBP were initiated during but persisted after the cessation of blue light stimulation, a time course that is suggestive of an endocrine mechanism (Yosten et al., 2011). Heart rate was unchanged by the stimulation protocol (Fig. $4 E$; interaction was not significant; $\left.F_{(55,385)}=1.2 ; p=0.22\right)$.

In addition to cardiovascular responses, we evaluated drinking behavior to determine whether the activity of ATlaR neurons in the MnPO/OVLT influences body fluid homeostasis by controlling water and sodium consumption. Blue light stimulation robustly increased intake of water $\left(t_{(14)}=2.9 ; p=0.011\right)$ and $0.3 \mathrm{M} \mathrm{NaCl}\left(t_{(14)}=2.3 ; p=0.035\right)$ when need-free mice were given access to both (Fig. $4 F, G$ ). In these same mice, overnight water restriction increased the intake of water and $0.3 \mathrm{M} \mathrm{NaCl}$; however, blue light stimulation did not further increase intake, suggesting that this particular pattern of optogenetic stimulation may elicit a maximal drinking response. Importantly, AAVeYFP mice did not exhibit alterations in water or $0.3 \mathrm{M} \mathrm{NaCl}$ intake in response to blue light stimulation under need-free (water: $33 \pm 6.0$ vs $26 \pm 3.5$ licks/ 10 min; $0.3 \mathrm{M} \mathrm{NaCl}: 32 \pm 4.9$ vs $34 \pm 4.8$ licks $/ 10 \mathrm{~min}$ ) or deprived conditions (water: $142 \pm$ 33 vs $77 \pm 23$ licks/ $10 \mathrm{~min}$; $0.3 \mathrm{M} \mathrm{NaCl}: 149 \pm 45$ vs $190 \pm 49$ licks/10 min).

In a separate study, we examined whether inhibition of AT1aR neurons of the MnPO/OVLT using halorhodopsin affects cardiovascular parameters or fluid intake. For these experiments, we first conducted in vitro whole-cell patch-clamp recordings to confirm the ability of continuous application of laser light to silence AT1aR neurons (Fig. $4 H-J$ ). Interestingly, this same continuous inhibition of ATlaR neurons in the MnPO/OVLT over a period of $10 \mathrm{~min}$ results in a significant reduction in SBP (Fig. $4 K$; significant interaction; $F_{(24,192)}=2.3 ; p=0.0009$ ); however, no effects on heart rate were observed (Fig. $4 L$; interaction was not significant; $\left.F_{(24,192)}=0.79 ; p=0.75\right)$. Finally, we used optogenetic inhibition to determine whether ATlaR neurons in the MnPO/OVLT are necessary for need-free and/or deprivation-induced water and sodium consumption. As can be seen in 

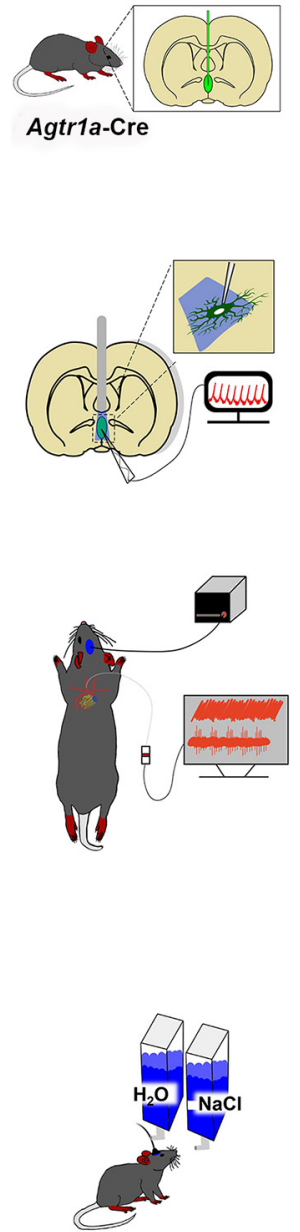
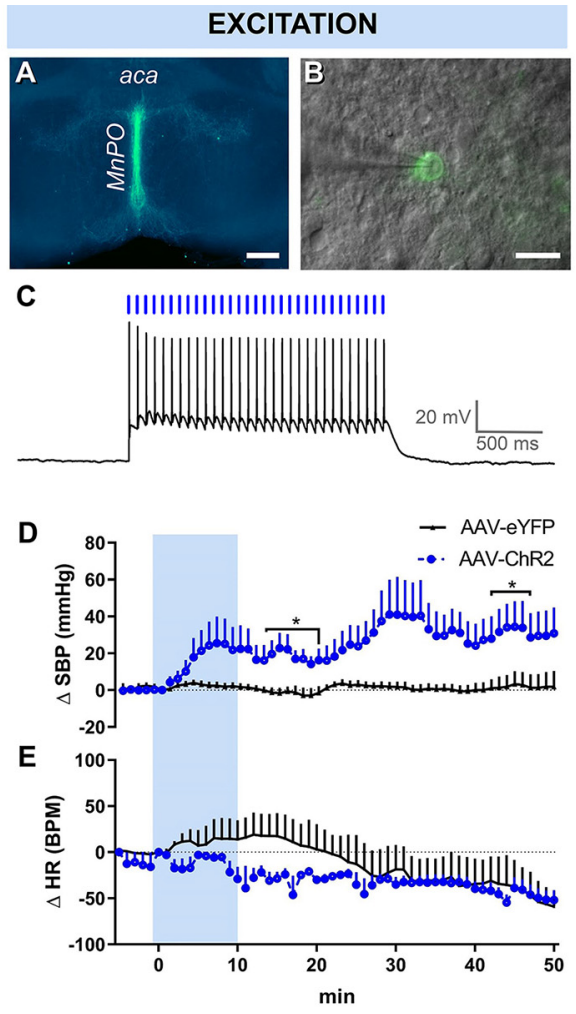

$\mathbf{F}$

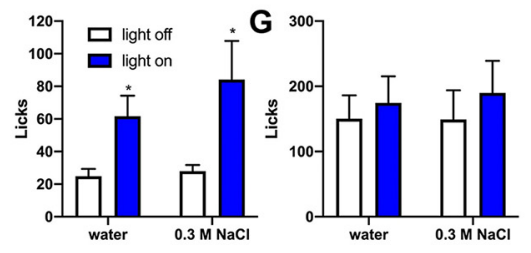

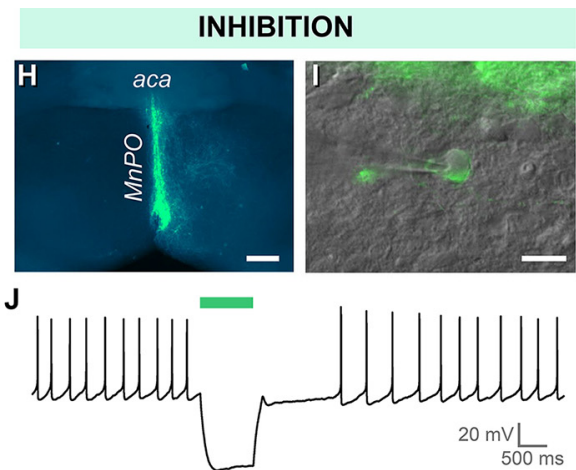

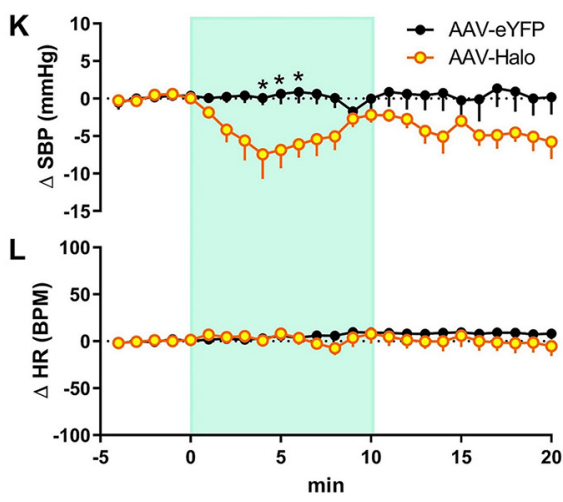

M
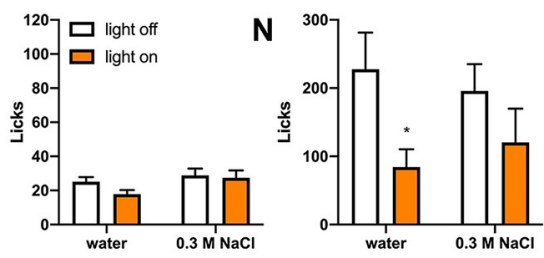

Figure 4. Optogenetic stimulation of AT1aR-containing neurons in the MnP0/OVLT influences blood pressure. $\boldsymbol{A}$, Cre-dependent expression of ChR2/eYFP (green) in MnP0/OVLT of an Agtr7a-Cre mouse. $\boldsymbol{B}, \mathrm{A}$ combination of epifluorescence and DIC microscopy was used to target virally transformed neurons for study. $\boldsymbol{C}$, Exposure to $15 \mathrm{~Hz}$ blue light produces action potentials. $D, S B P$ and $(E)$ heart rate (HR) response to blue light stimulation of AT1aR neurons residing in the MnPO/OVLT (10 mW; $15 \mathrm{~Hz} ; 20 \mathrm{~ms}$ pulse width; $60 \mathrm{~s}$ 0N/OFF; performed over a period of $10 \mathrm{~min}, n=4$ or $5 /$ group). Water and $0.3 \mathrm{~m} \mathrm{NaCl}$ intake in response to the same stimulation parameters $(\boldsymbol{F})$ during free access to water and $(\boldsymbol{G})$ subsequent to $16 \mathrm{~h}$ of water deprivation; $n=$ 15 mice. $\boldsymbol{H}$, Cre-dependent expression of halorhodopsin (Halo)/eYFP (green) in the MnPO/OVLT of an AT1aR-Cre mouse. $\boldsymbol{I}$, A combination of epifluorescence and DIC microscopy was used to target virally transformed neurons for study. $J$, Green light hyperpolarizes AT1aR neurons in the MnP0/OVLT. Changes in $(\boldsymbol{K})$ SBP and $(\boldsymbol{L})$ HR in response to optogenetic inhibition of AT1aR neurons of the MnPO/OVLT in mice receiving AAV-Halo; $n=6 /$ group. Water and $0.3 \mathrm{~m} \mathrm{NaCl}$ intake in response to optogenetic inhibition $(\boldsymbol{M})$ during free access to water and $(\boldsymbol{M})$ subsequent to $16 \mathrm{~h}$ of water deprivation; $n=9$ mice. aca, anterior commissure. Error bars indicate SEM. $* p<0.05$. Scale bars: $\boldsymbol{A}, \boldsymbol{H}, 250 \mu \mathrm{m} ; \boldsymbol{B}, \boldsymbol{I}, 20 \mu \mathrm{m}$.

Figure $4 M$, inhibition of ATlaR neurons in the MnPO/OVLT had no effect on need-free intake of water and $0.3 \mathrm{M} \mathrm{NaCl}$. Conversely, mice receiving the AAV-Halo drank significantly less water following overnight water deprivation during light stimulation compared with the Light off control condition (Fig. $4 N$, left; $\left.t_{(9)}=2.9 ; p=0.02\right)$. However, this suppression of intake appears specific to water because optogenetic inhibition had no effect on consumption of $0.3 \mathrm{M} \mathrm{NaCl}$ (Fig. $4 \mathrm{~N}$, right).

\section{Optogenetic excitation of AT1aR-expressing neurons in the MnPO/OVLT elicits fos induction in AVP neurons in the PVN}

Next, we explored potential mechanism(s) underlying the increased blood pressure that accompanied optogenetic excitation of AT1aR neurons in the MnPO/OVLT. Initial experiments centered on whether forebrain nuclei found to contain axons originating from AT1aR neurons in the MnPO/OVLT were activated subsequent to optogenetic excitation. Toward this end, Agtr1a-Cre mice were delivered AAV-ChR2 or AAV-eYFP into the MnPO/OVLT and implanted with a chronic dwelling fiber- optic as previously described. Following surgical recovery and habituation to the fiber-optic tether, blue light was delivered to the $\mathrm{MnPO} / \mathrm{OVLT}$ and $90 \mathrm{~min}$ later, mice were perfused and brain sections through the MnPO/OVLT, PVN, and SON of the hypothalamus were processed for immunohistochemistry to label Fos, a marker of neuronal activation. As expected, optogenetic excitation of AT1aR neurons within the MnPO/OVLT increased the number of Fos-immunoreactive nuclei observed within the MnPO and OVLT themselves (Fig. 5A-D). Triplelabel immunohistochemistry revealed that optogenetic excitation of ATlaR neurons in the MnPO/OVLT was associated with increased Fos in AVP-synthesizing neurons of the SON (Fig. $5 E, F$ ) and PVN (Fig. 5G-J). Intriguingly, eYFP-labeled fibers arising from AT1aR neurons within the MnPO/OVLT appear to make appositions onto neurons within the PVN that were double-labeled for Fos and AVP (Fig. 5I,J). Quantitative analyses of these results are shown in Figure $5 K, L$ and confirmed that the total number of Fos-positive nuclei was indeed elevated in the MnPO/OVLT (Fig. $5 K$; $t_{(9.56)}=3.5 ; p=0.006$ ). Although the absolute number of Fos-positive nuclei within the SON and PVN were 
OVLT
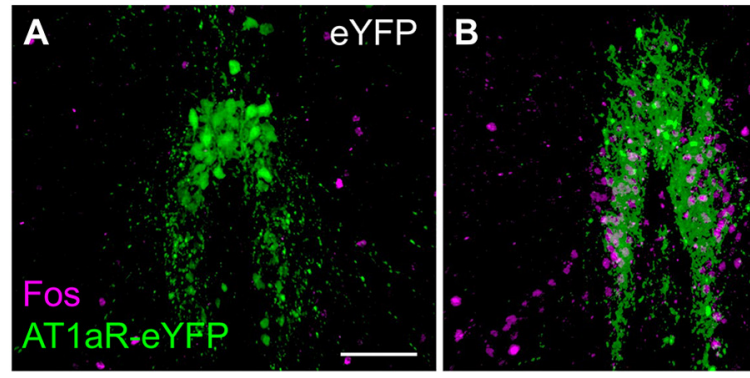

ChR2
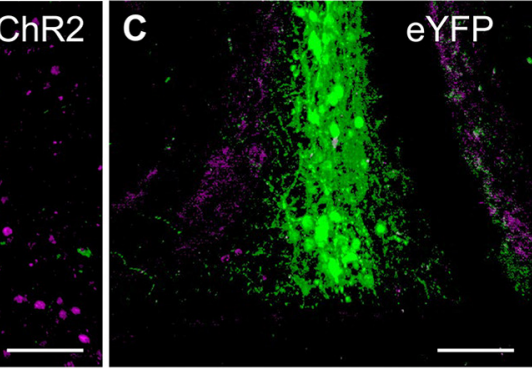

$\mathrm{MnPO}$

SON
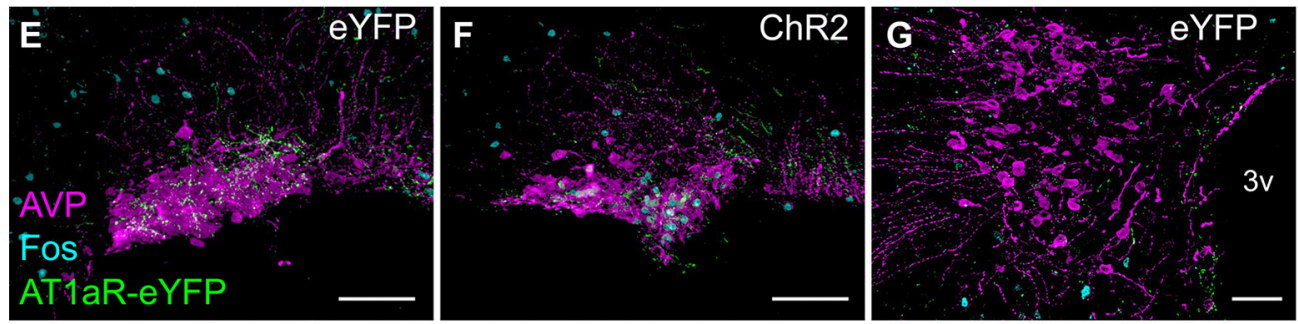

PVN
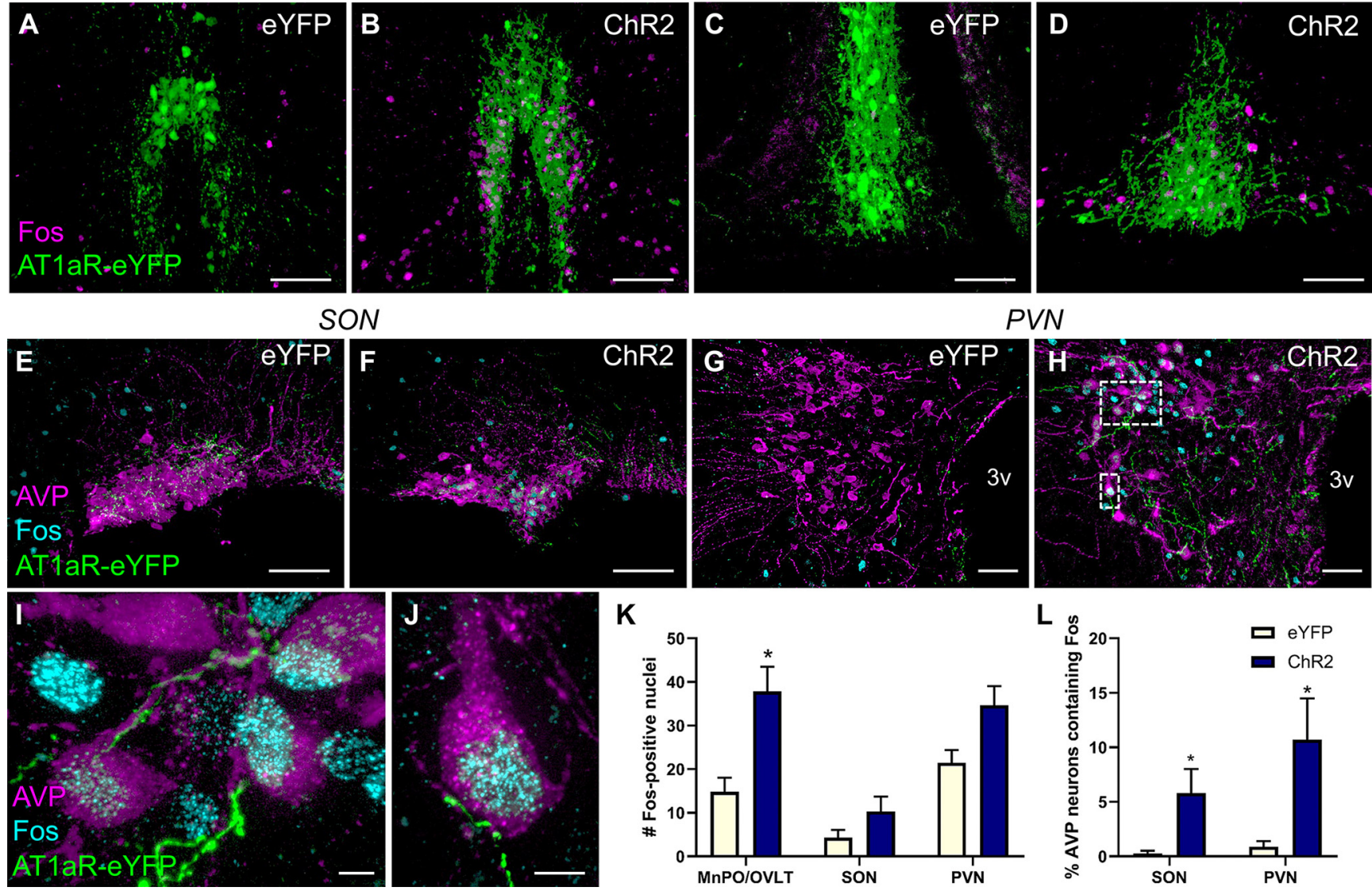

K

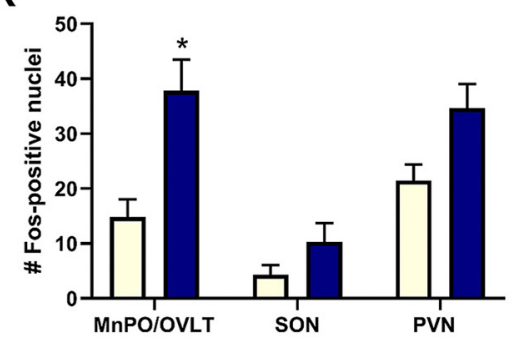

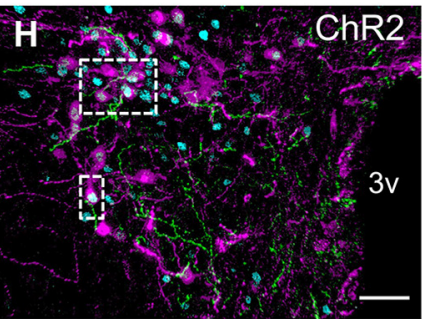

$\mathbf{L}$

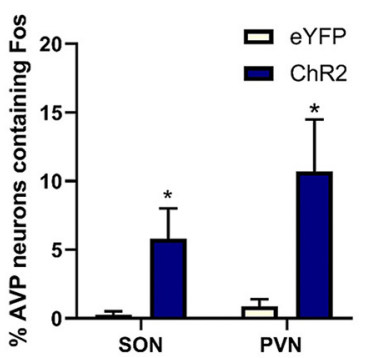

Figure 5. Stimulation of AT1aR-containing neurons within the MnP0/OVLT activates neurons in the PVN and SON that synthesize AVP. Control mice (eYFP) and mice receiving the Cre-dependent AAV for the expression the ChR2 and eYFP were subjected to the same optogenetic stimulation protocol as in Figure 4. Ninety minutes after the onset of optogenetic stimulation, mice were killed and perfused, and brains were processed for Fos immunoreactivity. Representative projection images through the $0 \mathrm{VLT}$ and $\mathrm{MnPO}$ of $(\boldsymbol{A}, \boldsymbol{C})$ an eYFP control and $(\boldsymbol{B}, \boldsymbol{D})$ a $\mathrm{ChR2}$ mouse subjected to optogenetic stimulation. Magenta represents Fos. Green represents AT1aR-expressing neurons. Representative projection images through the SON and PVN of an ( $\boldsymbol{E}, \mathbf{G})$ eYFP control and a $(\boldsymbol{F}, \boldsymbol{H}$ - J) ChR2 mouse depicting AT1aR-eYFP neuronal fibers (green) arising from the MnP0/OVLT, AVP (magenta), and Fos immunoreactivity (cyan). $\boldsymbol{K}$, Total Fos-positive nuclei/section in the brain ROls and $(\boldsymbol{L})$ the percentage of AVP neurons containing Fos within the SON and PVN. Error bars indicate SEM. $n=7 /$ group. $* p<0.05$. Scale bars: $\boldsymbol{A}-\boldsymbol{F}, 100 \mu \mathrm{m} ; \boldsymbol{G}, \boldsymbol{H}, 50 \mu \mathrm{m} ; \boldsymbol{I}, \boldsymbol{J}$, $5 \mu \mathrm{m}$.

not significantly different between the groups, optogenetic stimulation of ATlaR neurons in the MnPO/OVLT resulted in a significantly greater percentage of AVP neurons that expressed Fos in AAV-ChR2 mice relative to mice given the AAV-eYFP control virus (Fig. $5 L$; main effect of group; $F_{(1.25)}=13.67 ; p=0.001$ ).

\section{Optogenetic excitation of afferents arising from AT1aR neurons in the MnPO/OVLT drives glutamatergic} neurotransmission onto AVP neurons in the PVN

Based on the results thus far, we hypothesized that AT1aR neurons in the MnPO/OVLT form excitatory synapses onto neurons in the PVN that increase blood pressure by promoting the release of AVP into the systemic circulation. To test this hypothesis, an additional cohort of mice received injections of Cre-dependent AAV-ChR2 in the MnPO/OVLT. After allowing 2-3 weeks for viral transduction, brain slices were made through the PVN and used for in vitro whole-cell patch-clamp electrophysiological recordings. Magnocellular neurons were identified by their large soma (Fig. $6 B$ ), and also by the presence of a prominent outward rectifying potassium current $\left(\mathrm{I}_{\mathrm{A}}\right)$, which produces a long delay to the first action potential during direct current injection after a hyperpolarizing prepulse (Fig. 6C) (Luther and Tasker, 2000). Once identified, PVN magnocellular neurons were voltageclamped at $-70 \mathrm{mV}$, and blue light was used to evoke transmitter release from axon terminals arising from ATlaR neurons residing in the MnPO/OVLT. In total, we found that optogenetic stimulation evoked a clear EPSC in 15 of the 65 PVN magnocellular neurons that were surveyed. In 5 of these 15 neurons, we then bath-applied the ionotropic glutamate receptor antagonists DNQX and APV and found that light-evoked EPSCs were effectively eliminated (peak amplitude after glutamate receptor antagonists was reduced to $8.8 \pm 2.7 \%$ of baseline, $n=5, p=0.003$; Fig. $6 D)$. In 4 of the 15 light-responsive PVN magnocellular neurons, we fixed the tissue after whole-cell recordings were complete, and then performed IHC for vasopressin-neurophysin and fluorescently labeled biocytin to further determine whether the lightresponsive neurons were likely vasopressinergic. Consistent with that hypothesis, all four such neurons examined were immunoreactive for vasopressin-neurophysin (e.g., Fig. $6 E$ ). While completing these studies, we also tested for optogenetic stimulation evoked EPSCs in parvocellular PVN neurons $(n=11)$ that lacked and $\mathrm{I}_{\mathrm{A}}$ but exhibited a low threshold spike, a common characteristic of preautonomic neurons with direct projections to the RVLM that control sympathetic outflow to cardiovascular tissues (Luther and Tasker, 2000; Luther et al., 2002). We also recorded from an additional $18 \mathrm{PVN}$ neurons that lacked both $\mathrm{I}_{\mathrm{A}}$ and low threshold spike, a phenotype observed in corticotrophin-releasing hormone neurosecretory neurons (Wamsteeker Cusulin et 
A
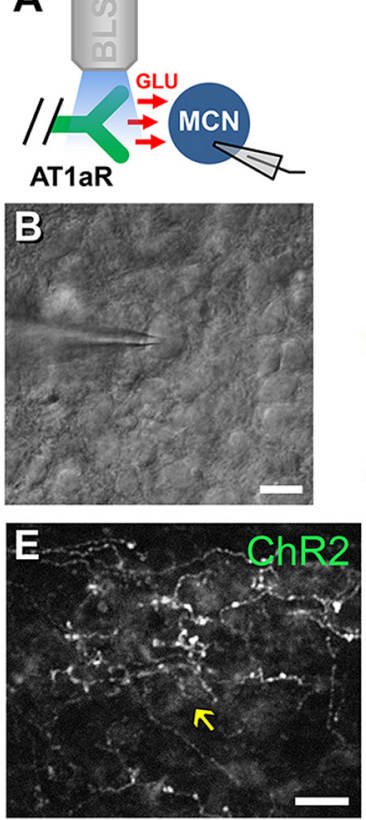

F

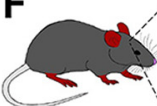

Agtr1a-Cre
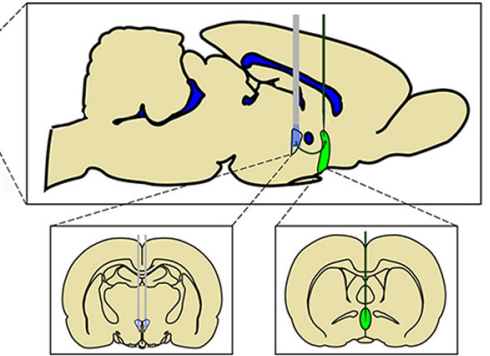

R
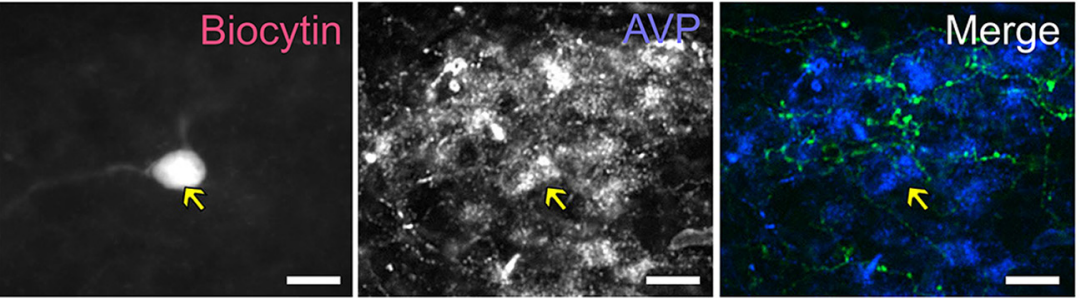

G

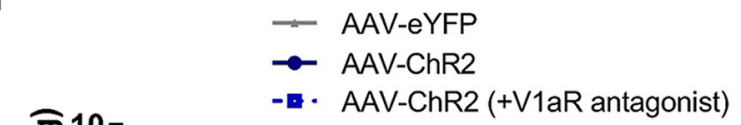

올

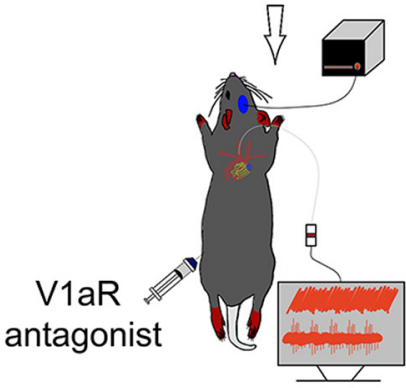

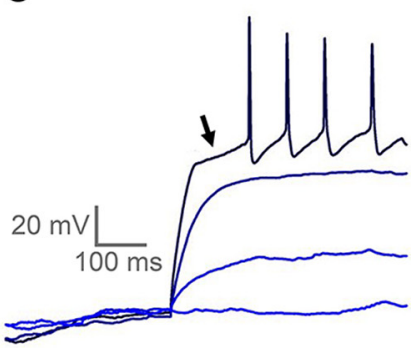

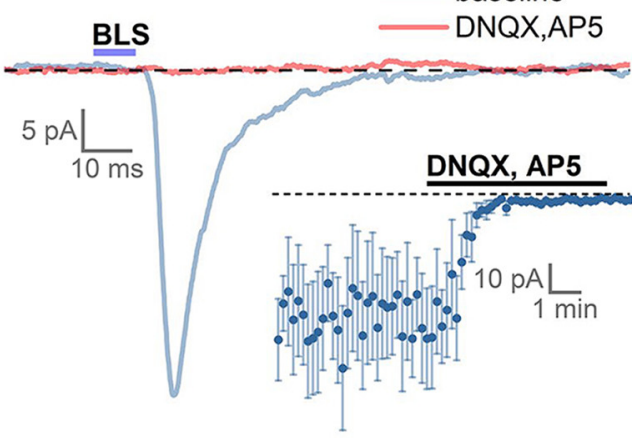

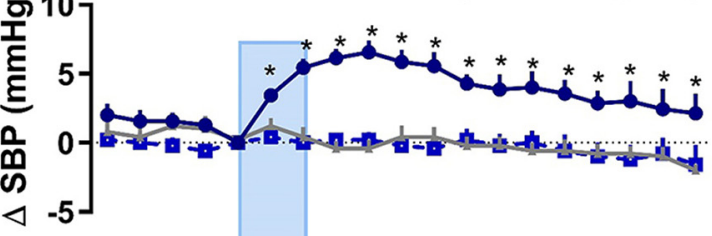

H

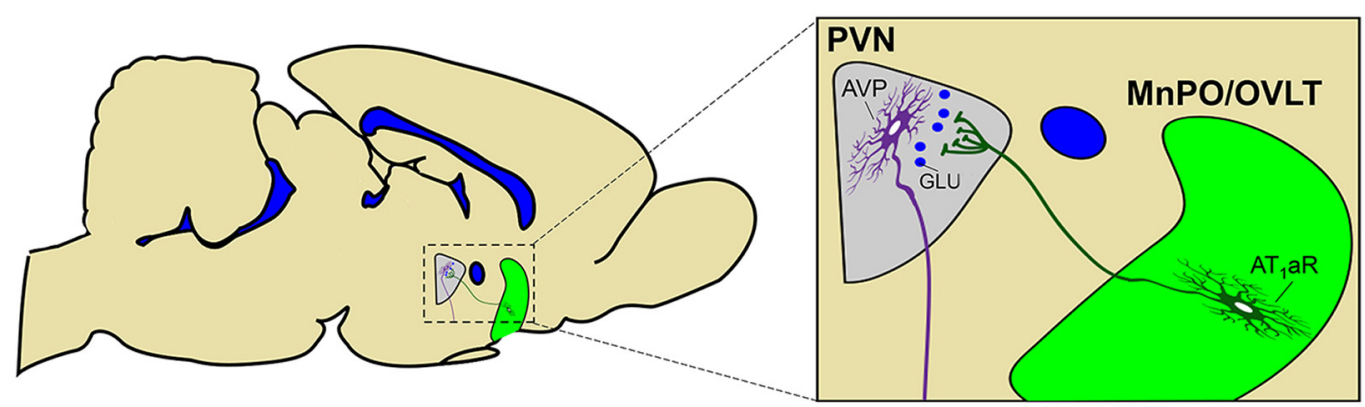

Figure 6. Optogenetic stimulation of axons in the PVN that originate from MnP0/OVLT AT1aR neurons releases glutamate (GLU) onto magnocellular (MCN) PVN neurons and induces a V1aR-dependent elevation in blood pressure. $\boldsymbol{A}$, Experimental design for in vitro optogenetic study. B, PVN magnocellular neurons were initially targeted based on their location, large soma, and general morphology as apparent under IR-DIC. C, Classification as a PVN magnocellular neuron was confirmed during current-clamp recordings based on the presence of a large $I_{A}$ current. This current is responsible for the long delay to first action potential observed in response to suprathreshold stimulation following a brief hyperpolarizing step (arrow). D, Blue light stimulation (BLS) evoked EPSCs in 15 of 65 magnocellular neurons tested. A subset of light-evoked responses observed (5 of 15) were challenged with ionotropic glutamate receptor antagonists (DNQX and AP5), which effectively eliminated the response (inset). $\boldsymbol{E}$, Post hoc immunohistochemical assessment of a light-responsive PVN magnocellular neuron suggests an AVP phenotype. $\boldsymbol{F}$, Schematic depicting the experimental design used to determine the role of V1aR in cardiovascular responses to blue light stimulation of afferents arising from AT1aR-containing neurons of the MnPO/OVLT. For these studies, the AAV-ChR2 or AAV-eYFP was injected into the MnP0/OVLT of Agtr1a-Cre mice and the fiber-optic post was positioned over the PVN. G, SBP (top) and HR (bottom) response to blue light stimulation of axons in the PVN arising from MnP0/OVLT AT1aR-expressing neurons (10 mW; $15 \mathrm{~Hz} ; 60 \mathrm{~s}$ ). $n=4$ or 5/group. $\boldsymbol{H}$, lllustration of the overall conclusion that AT1aR neurons in the MnP0/OVLT elicits elevations in blood pressure, in part, via excitatory connections to neurons in the PVN that secrete AVP into the systemic circulation. Error bars indicate SEM. $* p<0.05$. Scale bars, $20 \mu \mathrm{m}$. 
al., 2013; Pati et al., 2020). Notably, none of these 29 neurons exhibited an EPSC in response to blue light. Given the prominent role that the MnPO/OVLT plays in the autonomic control of blood pressure (Ployngam and Collister, 2008; Llewellyn et al., 2012; de Lima Silveira et al., 2014; Kinsman et al., 2017), followup experiments further evaluated the possibility that AT1aR neurons have direct excitatory projections to preautonomic neurons. We delivered AAV-ChR2 into the MnPO/OVLT of Agtr1a-Cre mice as done previously but also administered a retrograde tracer (AAVrg-CAG-tdTomato) into the RVLM, thereby allowing visualization of preautonomic neurons in the PVN. We found that 28 of the 32 retrogradely labeled neurons exhibited the expected low threshold spike; however, 0 of 32 demonstrated an EPSC in response to blue light stimulation. Overall, these results indicate that neurons in the MnPO/OVLT that express the ATlaR send excitatory glutamatergic projections to PVN which target the vasopressinergic neurons that are responsible for the hormone's secretion into the systemic circulation.

\section{Optogenetic excitation of MnPO/OVLT-arising AT1aR afferents in the PVN is sufficient to elevate blood pressure and this effect is abolished in the presence of a V1aR antagonist}

The results from our previous experiments suggest that Ang-II excites neurons in the MnPO/OVLT that have direct glutamatergic projections onto neurons in the PVN that elevate blood pressure by driving the systemic release of AVP. To directly test this hypothesis, we again administered Agtrla-Cre mice the AAV-ChR2 or the AAV-eYFP control into the MnPO/OVLT; however, this time, the fiber-optic was positioned directly dorsal to the PVN (Fig. $6 F$ ) such that axons originating from these neurons could specifically be targeted for optogenetic stimulation during anesthetized cardiovascular recordings. As can be seen in Figure $6 G$, in vivo optogenetic excitation of axons in the PVN arising from ATlaR-expressing neurons increases SBP in AAV-ChR2 mice relative to AAV-eYFP controls without significantly impacting heart rate. Qualitative comparison between these data and those depicted in Figure 4 suggest, however, that the stimulation of these axon terminals may be of a lesser magnitude and duration than that which is observed subsequent to blue light stimulation of the soma of ATlaR neurons residing within the MnPO/OVLT. This reduced magnitude and duration may be because of the abbreviated time course of optical stimulation that was used to efficiently combine pharmacology with optogenetics. On the other hand, it may relate to a difference in the ability of blue light stimulation of axons versus soma to lead to neurotransmitter release. Finally, it may also suggest that projections from ATlaR neurons residing within the MnPO/OVLT to other brain areas, such as the SON or $\mathrm{DMH}$, may contribute to the pressor actions of these neurons. Nonetheless, and consistent with our hypothesis that the pressor effect is mediated by excitation of vasopressinergic PVN neurons, this increase in SBP was completely abolished by systemic pretreatment with a $\mathrm{VlaR}$ antagonist. In other words, optogenetic stimulation of these afferents no longer induced a pressor response in mice that were pretreated with the V1aR antagonist. Together, these results indicate that activation of ATlaR neurons in the MnPO/OVLT elicits elevations in blood pressure, in part, via excitatory connections to neurons in the PVN that secrete AVP into the systemic circulation (Fig. 6H).

\section{Discussion}

These studies used a multifaceted approach to evaluate the structure and function of neurons in the lamina terminalis $(\mathrm{MnPO} /$
OVLT) that express ATlaR(s). We found that these neurons are predominantly glutamatergic, are excited by Ang-II, and send axonal projections to brain nuclei regulating fluid intake, AVP release, and blood pressure. Consistent with this connectivity, in vivo optogenetic excitation of ATlaR neurons in the $\mathrm{MnPO} /$ OVLT causes large and sustained elevations in blood pressure and impacts water and sodium consumption, whereas their optogenetic inhibition produces opposite effects. Neuroanatomical and electrophysiological studies revealed that vasopressinergic PVN neurons receive excitatory glutamatergic projections from AT1aR neurons in the MnPO/OVLT, thereby suggesting a contributing role for AVP in the pressor actions of these neurons. In support of this causality, in vivo optogenetic excitation of axons in the PVN that originate from these neurons produces an increase in blood pressure, which is abolished by systemic delivery of a V1aR antagonist. Collectively, these results demonstrate that excitation of ATlaR neurons in the MnPO/OVLT influences fluid intake, AVP release, and blood pressure. The implication is that dysregulation of this circuit may contribute to cardiovascular disorders, such as hypertension and congestive heart failure.

The tissue surrounding the anteroventral third ventricle which encompasses the MnPO/OVLT has long been implicated in responses that maintain body fluid homeostasis. Early studies determined that administration of Ang-II or hypertonic saline into the area elicits drinking (Buggy et al., 1975), while lesion of these nuclei produces thirst deficits and hypernatremia (Johnson and Buggy, 1978). Here, we demonstrate that ATlaR neurons in the MnPO/OVLT are activated by Ang-II and that their optogenetic excitation promotes, but their inhibition attenuates, drinking in mice, results that are further supportive of a role for these areas in body fluid homeostasis. The MnPO/OVLT are comprised of glutamatergic and GABAergic neurons with each phenotype having extensive projections throughout the brain (McKinley et al., 2015; Abbott et al., 2016). These studies reveal that ATlaR neurons in the area are mostly glutamatergic and appear to have more targeted projections. Despite these limited projections, optogenetic excitation of these ATlaR neurons elicits water intake that is comparable to what is observed with optogenetic excitation of all glutamate neurons in the area (Abbott et al., 2016). These collective results suggest that ATlaR demarcates specific neurons in the MnPO/OVLT that drive water consumption.

In addition to promoting drinking, $\mathrm{AT} 1 \mathrm{aR}(\mathrm{s})$ within the brain control blood pressure via the systemic release of neuroendocrine factors, like AVP, that promote vasoconstriction and renal reabsorption of water, and also by adjusting sympathetic outflow to cardiovascular tissues. The PVN is an integrative nucleus that contains neurosecretory and preautonomic neurons that regulate AVP release and sympathetic outflow, respectively. Because this area also robustly expresses AT1aRs, it is reasonable to hypothesize that Ang-II-induced activation of PVN neurons that express ATlaR drives AVP release and sympathetic outflow to increase blood pressure. This hypothesis is complicated, however, by data indicating that AVP and preautonomic neurons in the PVN do not themselves express ATlaRs, but are instead innervated by ATlaR-expressing neurons that reside in brain regions with projections to the PVN (de Kloet et al., 2017). Thus, it is possible that stimulation of AT1aRs residing on neurons outside the PVN produces elevations in blood pressure via excitatory connections onto neurons controlling AVP release and sympathetic outflow.

Our studies delve deeply into a neural circuit that involved the PVN and contributes to the potent influence that central AT1aRs have on blood pressure. Along these lines, the anteroventral third 
ventricle region also participates in cardiovascular regulation. Lesion of the area blunts elevations in blood pressure and AVP release that accompany central delivery of Ang-II (Johnson et al., 1978; Bealer et al., 1979). Furthermore, pharmacological or optogenetic excitation of certain neurons in the area acutely elevates blood pressure (Ployngam and Collister, 2007; Llewellyn et al., 2012; Leib et al., 2017). Here, we find that optogenetic inhibition of AT1aR neurons in the MnPO/OVLT lowers blood pressure, whereas optogenetic excitation evokes large increases in blood pressure. Interestingly, these elevations in blood pressure were sustained, a time course that is suggestive of an underlying endocrine contribution. To probe for potential mechanisms of these cardiovascular effects, we combined neuroanatomical tract tracing with optogenetics to reveal that excitation of ATlaR neurons in the MnPO/OVLT elicits Fos induction within AVP neurons in the SON and PVN. Furthermore, our electrophysiological experiments revealed that AT1aR neurons in the MnPO/OVLT form glutamatergic synapses with PVN neurons that exhibit electrophysiological properties of magnocellular neurons (Hoffman et al., 1991) and express AVP-neurophysin-2. This confirms that at least a subset of structures observed in the PVN that arise from AT1aR neurons in the $\mathrm{MnPO} / \mathrm{OVLT}$ are functional synaptic terminals. With regard to the functionality of these connections, our final experiments revealed that in vivo optogenetic excitation of axon terminals in the PVN that originate from ATlaR-expressing neurons in the MnPO/OVLT increases blood pressure. Although this pressor response is of lesser magnitude than that which was observed when stimulating AT1aR neuronal cell bodies themselves in the MnPO/OVLT, due perhaps to the shorter time course of stimulation or to additional intricacies in neural circuitry, it was eliminated by systemic administration of a V1aR antagonist. These results are consistent with a causal role for this neural circuit in mediating a pressor response that is dependent on activation of V1aR.

In addition to being expressed on magnocellular neurons that project to the posterior pituitary, AVP is also synthesized by parvocellular PVN neurons that send afferents to the median eminence, RVLM, and spinal cord (Swanson et al., 1980; Shafton et al., 1998; Pyner and Coote, 2000). It is possible that activation of parvocellular neurons contributes to the increase in blood pressure that follows optogenetic excitation of AT1aR neurons in the MnPO/OVLT. To evaluate this possibility, we patch-clamped PVN neurons that exhibited electrophysiological properties of parvocellular preautonomic or neurosecretory neurons (Stern, 2001; Luther et al., 2002); however, optogenetic excitation did not elicit EPSCs in these neurons, suggesting that glutamatergic AT1aR neurons in the MnPO/OVLT do not synapse onto neurons in the PVN that control sympathetic outflow. It is established that MnPO projections into the PVN influence sympathetic outflow (Stocker and Toney, 2005; Llewellyn et al., 2012) and the absence of EPSCs in preautonomic neurons was unexpected. Consequently, we used retrograde neuronal tract tracing to visualize neurons in the PVN that project to the RVLM and probed for EPSCs during optogenetic activation of nerve terminals originating from ATlaR neurons in the MnPO/OVLT. Although the majority of neurons expressing the retrograde label had electrophysiological properties consistent with those of preautonomic neurons (Luther and Tasker, 2000; Luther et al., 2002), none exhibited EPSCs on optogenetic stimulation.

Our results demonstrate that $\mathrm{ATlaR}$ neurons in the $\mathrm{MnPO} /$ OVLT increase blood pressure by activating neurons in the hypothalamus that control the release of AVP; however, the mechanism underlying the excitatory influence of Ang-II on preautonomic neurons remains to be discerned. While we cannot definitively rule out that ATlaR neurons in the MnPO/OVLT excite preautonomic PVN neurons, our results suggest that an alternative mechanism(s) may underlie the sympathoexcitatory actions of central ATlaR. For example, Ang-II may act on ATlaRs expressed on neurons in the SFO with excitatory projections onto preautonomic PVN neurons (Z. Li and Ferguson, 1993; Krause et al., 2011). Alternatively, there is evidence that Ang-II acts presynaptically on ATlaR to reduce GABA release, thereby disinhibiting preautonomic PVN neurons (D. P. Li et al., 2003; D. P. Li and Pan, 2005). Crosstalk among the neuronal phenotypes residing within the PVN may also contribute to the increased blood pressure that accompanies activation of $\mathrm{AT} 1 \mathrm{aR}(\mathrm{s})$, and recent reports demonstrate paracrine signaling within the PVN resulting from dendritic release of neuropeptides (Son et al., 2013; Stern, 2015; Brown et al., 2020; Pati et al., 2020). Finally, it is likely that neurons in the MnPO/OVLT that express ATlaRs make connections beyond the PVN that mediate the autonomic and behavioral effects of Ang-II. Our anterograde neuronal tracing revealed fibers within several brain nuclei, including the periaqueductal gray, $\mathrm{DMH}$, and SFO, which are implicated in control of autonomic outflow and/or fluid intake (Augustine et al., 2018; Kono et al., 2020). Future studies examining these connections are necessary to provide a comprehensive view of how Ang-II acts in the brain to control body fluid and cardiovascular homeostasis.

Cardiovascular disorders, like hypertension and congestive heart failure, are accompanied by increased AVP release and hydromineral imbalance that can be ameliorated with treatments limiting AT1aR activation. Interestingly, lesion of the anteroventral third ventricle region reverses the development of experimentally induced-hypertension (Haywood et al., 1983). Recently, Marciante et al. (2020) determined that ablation of glutamatergic projections from the MnPO to the PVN also prevents development of hypertension that follows chronic intermittent hypoxia. This antihypertensive effect was recapitulated by selective knockdown of Agtrla within the MnPO (Marciante et al., 2019). These results, in conjunction with our own, suggest that neurons in the MnPO/OVLT that express the ATlaR may be specifically targeted to reduce AVP release and blood pressure and may therefore serve as a target for therapeutics that combat the onset of hypertension and congestive heart failure.

\section{References}

Abbott SB, Machado NL, Geerling JC, Saper CB (2016) Reciprocal control of drinking behavior by median preoptic neurons in mice. J Neurosci 36:8228-8237.

Augustine V, Gokce SK, Lee S, Wang B, Davidson TJ, Reimann F, Gribble F, Deisseroth K, Lois C, Oka Y (2018) Hierarchical neural architecture underlying thirst regulation. Nature 555:204-209.

Bealer SL, Phillips MI, Johnson AK, Schmid PG (1979) Anteroventral third ventricle lesions reduce antidiuretic responses to angiotensin II. Am J Physiol 236:E610-E615.

Ben-Barak Y, Russell JT, Whitnall MH, Ozato K, Gainer H (1985) Neurophysin in the hypothalamo-neurohypophysial system: I. Production and characterization of monoclonal antibodies. J Neurosci 5:81-97.

Brown CH, Ludwig M, Tasker JG, Stern JE (2020) Somato-dendritic vasopressin and oxytocin secretion in endocrine and autonomic regulation. J Neuroendocrinol 32:e12856.

Buggy J, Fisher AE (1976) Anteroventral third ventricle site of action for angiotensin induced thirst. Pharmacol Biochem Behav 4:651-660.

Buggy J, Fisher AE, Hoffman WE, Johnson AL, Phillips MI (1975) Ventricular obstruction: effect on drinking induced by intracranial injection of angiotensin. Science 190:72-74. 
Cato MJ, Toney GM (2005) Angiotensin II excites paraventricular nucleus neurons that innervate the rostral ventrolateral medulla: an in vitro patch-clamp study in brain slices. J Neurophysiol 93:403-413.

Chiodera P, Volpi R, Caiazza A, Giuliani N, Magotti MG, Coiro V (1998) Arginine vasopressin and oxytocin responses to angiotensin II are mediated by AT1 receptor subtype in normal men. Metabolism 47:893-896.

Constantinides C, Angeli S, Mean R (2011a) Murine cardiac hemodynamics following manganese administration under isoflurane anesthesia. Ann Biomed Eng 39:2706-2720.

Constantinides C, Mean R, Janssen BJ (2011b) Effects of isoflurane anesthesia on the cardiovascular function of the C57BL/6 mouse. ILAR J 52:e21e31.

Coote JH, Yang Z, Pyner S, Deering J (1998) Control of sympathetic outflows by the hypothalamic paraventricular nucleus. Clin Exp Pharmacol Physiol 25:461-463.

de Kloet AD, Wang L, Ludin JA, Smith JA, Pioquinto DJ, Hiller H, Steckelings UM, Scheuer DA, Sumners C, Krause EG (2016) Reporter mouse strain provides a novel look at angiotensin Type 2 receptor distribution in the central nervous system. Brain Struct Funct 221:891-912.

de Kloet AD, Wang L, Pitra S, Hiller H, Smith JA, Tan Y, Nguyen D, Cahill KM, Sumners C, Stern JE, Krause EG (2017) A unique "angiotensin-sensitive" neuronal population coordinates neuroendocrine, cardiovascular, and behavioral responses to stress. J Neurosci 37:3478-3490.

de Lima Silveira L, da Silva EF, de Andrade AM, Xavier CH, Freiria-Oliveira AH, Colugnati DB, de Castro CH, Colombari E, Pedrino GR (2014) Involvement of the median preoptic nucleus in blood pressure control. Neurosci Lett 558:91-96.

Edelstein A, Amodaj N, Hoover K, Vale R, Stuurman N (2010) Computer control of microscopes using microManager. Curr Protoc Mol Biol Chapter 14:Unit14.20.

Faulk K, Shell B, Nedungadi TP, Cunningham JT (2017a) Role of angiotensin-converting enzyme 1 within the median preoptic nucleus following chronic intermittent hypoxia. Am J Physiol Regul Integr Comp Physiol 312:R245-R252.

Faulk KE, Nedungadi TP, Cunningham JT (2017b) Angiotensin converting enzyme 1 in the median preoptic nucleus contributes to chronic intermittent hypoxia hypertension. Physiol Rep 5:e13277.

Haywood JR, Fink GD, Buggy J, Boutelle S, Johnson AK, Brody MJ (1983) Prevention of two-kidney, one-clip renal hypertension in rat by ablation of AV3V tissue. Am J Physiol 245:H683-H689.

Hoffman NW, Tasker JG, Dudek FE (1991) Immunohistochemical differentiation of electrophysiologically defined neuronal populations in the region of the rat hypothalamic paraventricular nucleus. J Comp Neurol 307:405-416.

Janssen BJ, De Celle T, Debets JJ, Brouns AE, Callahan MF, Smith TL (2004) Effects of anesthetics on systemic hemodynamics in mice. Am J Physiol Heart Circ Physiol 287:H1618-H1624.

Jessberger S, Toni N, Clemenson GD Jr, Ray J, Gage FH (2008) Directed differentiation of hippocampal stem/progenitor cells in the adult brain. Nat Neurosci 11:888-893.

Johnson AK, Buggy J (1978) Periventricular preoptic-hypothalamus is vital for thirst and normal water economy. Am J Physiol 234:R122-R129.

Johnson AK, Hoffman WE, Buggy J (1978) Attenuated pressor responses to intracranially injected stimuli and altered antidiuretic activity following preoptic-hypothalamic periventricular ablation. Brain Res 157:161-166.

Kádár A, Sánchez E, Wittmann G, Singru PS, Füzesi T, Marsili A, Larsen PR, Liposits Z, Lechan RM, Fekete C (2010) Distribution of hypophysiotropic thyrotropin-releasing hormone (TRH)-synthesizing neurons in the hypothalamic paraventricular nucleus of the mouse. J Comp Neurol 518: 3948-3961.

Kinsman BJ, Simmonds SS, Browning KN, Stocker SD (2017) Organum vasculosum of the lamina terminalis detects $\mathrm{NaCl}$ to elevate sympathetic nerve activity and blood pressure. Hypertension 69:163-170.

Kono Y, Yokota S, Fukushi I, Arima Y, Onimaru H, Okazaki S, Takeda K, Yazawa I, Yoshizawa M, Hasebe Y, Koizumi K, Pokorski M, Toda T, Sugita K, Okada Y (2020) Structural and functional connectivity from the dorsomedial hypothalamus to the ventral medulla as a chronological amplifier of sympathetic outflow. Sci Rep 10:13325.

Krause EG, de Kloet AD, Scott KA, Flak JN, Jones K, Smeltzer MD, UlrichLai YM, Woods SC, Wilson SP, Reagan LP, Herman JP, Sakai RR (2011) Blood-borne angiotensin II acts in the brain to influence behavioral and endocrine responses to psychogenic stress. J Neurosci 31:15009-15015.
Leib DE, Zimmerman CA, Poormoghaddam A, Huey EL, Ahn JS, Lin YC, Tan CL, Chen Y, Knight ZA (2017) The forebrain thirst circuit drives drinking through negative reinforcement. Neuron 96:1272-1281.e1274.

Lenkei Z, Corvol P, Llorens-Cortes C (1995) Comparative expression of vasopressin and angiotensin Type 1 receptor mRNA in rat hypothalamic nuclei: a double in situ hybridization study. Brain Res Mol Brain Res 34:135-142.

Lenkei Z, Palkovits M, Corvol P, Llorens-Cortès C (1997) Expression of angiotensin type-1 (AT1) and type-2 (AT2) receptor mRNAs in the adult rat brain: a functional neuroanatomical review. Front Neuroendocrinol 18:383-439.

Li DP, Pan HL (2005) Angiotensin II attenuates synaptic GABA release and excites paraventricular-rostral ventrolateral medulla output neurons. J Pharmacol Exp Ther 313:1035-1045.

Li DP, Chen SR, Pan HL (2003) Angiotensin II stimulates spinally projecting paraventricular neurons through presynaptic disinhibition. J Neurosci 23:5041-5049.

Li Z, Ferguson AV (1993) Subfornical organ efferents to paraventricular nucleus utilize angiotensin as a neurotransmitter. Am J Physiol Regul Integr Comp Physiol 265:R302-R309.

Llewellyn T, Zheng H, Liu X, Xu B, Patel KP (2012) Median preoptic nucleus and subfornical organ drive renal sympathetic nerve activity via a glutamatergic mechanism within the paraventricular nucleus. Am J Physiol Regul Integr Comp Physiol 302:R424-R432.

Loń S, Szczepańska-Sadowska E, Szczypaczewska M (1996) Evidence that centrally released arginine vasopressin is involved in central pressor action of angiotensin II. Am J Physiol 270:H167-H173.

Luther J, Tasker JG (2000) Voltage-gated currents distinguish parvocellular from magnocellular neurones in the rat hypothalamic paraventricular nucleus. J Physiol 523:193-209.

Luther JA, Daftary SS, Boudaba C, Gould GC, Halmos KC, Tasker JG (2002) Neurosecretory and non-neurosecretory parvocellular neurones of the hypothalamic paraventricular nucleus express distinct electrophysiological properties. J Neuroendocrinol 14:929-932.

Marciante AB, Wang LA, Farmer GE, Cunningham JT (2019) Selectively inhibiting the median preoptic nucleus attenuates angiotensin II and hyperosmotic-induced drinking behavior and vasopressin release in adult male rats. eNeuro 6:ENEURO.0473-18.2019.

Marciante AB, Wang LA, Little JT, Cunningham JT (2020) Caspase lesions of PVN-projecting MnPO neurons block the sustained component of $\mathrm{CIH}$-induced hypertension in adult male rats. Am J Physiol Heart Circ Physiol 318:H34-H48.

McKinley MJ, Yao ST, Uschakov A, McAllen RM, Rundgren M, Martelli D (2015) The median preoptic nucleus: front and centre for the regulation of body fluid, sodium, temperature, sleep and cardiovascular homeostasis. Acta Physiol (Oxf) 214:8-32.

Moffitt JR, Bambah-Mukku D, Eichhorn SW, Vaughn E, Shekhar K, Perez JD, Rubinstein ND, Hao J, Regev A, Dulac C, Zhuang X (2018) Molecular, spatial, and functional single-cell profiling of the hypothalamic preoptic region. Science 362:eaau5324.

Oldfield BJ, Davern PJ, Giles ME, Allen AM, Badoer E, McKinley MJ (2001) Efferent neural projections of angiotensin receptor (AT1) expressing neurones in the hypothalamic paraventricular nucleus of the rat. J Neuroendocrinol 13:139-146.

Pati D, Harden SW, Sheng W, Kelly KB, de Kloet AD, Krause EG, Frazier CJ (2020) Endogenous oxytocin inhibits hypothalamic corticotrophin-releasing hormone neurones following acute hypernatraemia. J Neuroendocrinol 32: e12839.

Pitra S, Worker CJ, Feng Y, Stern JE (2019) Exacerbated effects of prorenin on hypothalamic magnocellular neuronal activity and vasopressin plasma levels during salt-sensitive hypertension. Am J Physiol Heart Circ Physiol 317:H496-H504.

Ployngam T, Collister JP (2007) An intact median preoptic nucleus is necessary for chronic angiotensin II-induced hypertension. Brain Res 1162:6975.

Ployngam T, Collister JP (2008) Role of the median preoptic nucleus in chronic angiotensin II-induced hypertension. Brain Res 1238:75-84.

Pyner S, Coote JH (2000) Identification of branching paraventricular neurons of the hypothalamus that project to the rostroventrolateral medulla and spinal cord. Neuroscience 100:549-556.

Rowe BP, Grove KL, Saylor DL, Speth RC (1990) Angiotensin II receptor subtypes in the rat brain. Eur J Pharmacol 186:339-342. 
Schindelin J, Arganda-Carreras I, Frise E, Kaynig V, Longair M, Pietzsch T, Preibisch S, Rueden C, Saalfeld S, Schmid B, Tinevez JY, White DJ, Hartenstein V, Eliceiri K, Tomancak P, Cardona A (2012) Fiji: an opensource platform for biological-image analysis. Nat Methods 9:676-682.

Shafton AD, Ryan A, Badoer E (1998) Neurons in the hypothalamic paraventricular nucleus send collaterals to the spinal cord and to the rostral ventrolateral medulla in the rat. Brain Res 801:239-243.

Shi L, Guerra C, Yao J, Xu Z (2004) Vasopressin mechanism-mediated pressor responses caused by central angiotensin II in the ovine fetus. Pediatr Res 56:756-762.

Son SJ, Filosa JA, Potapenko ES, Biancardi VC, Zheng H, Patel KP, Tobin VA, Ludwig M, Stern JE (2013) Dendritic peptide release mediates interpopulation crosstalk between neurosecretory and preautonomic networks. Neuron 78:1036-1049.

Stern JE (2001) Electrophysiological and morphological properties of pre-autonomic neurones in the rat hypothalamic paraventricular nucleus. J Physiol 537:161-177.

Stern JE (2015) Neuroendocrine-autonomic integration in the paraventricular nucleus: novel roles for dendritically released neuropeptides. J Neuroendocrinol 27:487-497.

Stocker SD, Toney GM (2005) Median preoptic neurones projecting to the hypothalamic paraventricular nucleus respond to osmotic, circulating Ang II and baroreceptor input in the rat. J Physiol 568:599-615.

Swanson LW, Sawchenko PE (1980) Paraventricular nucleus: a site for the integration of neuroendocrine and autonomic mechanisms. Neuroendocrinology 31:410-417.
Swanson LW, Sawchenko PE, Wiegand SJ, Price JL (1980) Separate neurons in the paraventricular nucleus project to the median eminence and to the medulla or spinal cord. Brain Res 198:190-195.

Szczesny G, Veihelmann A, Massberg S, Nolte D, Messmer K (2004) Longterm anaesthesia using inhalatory isoflurane in different strains of micethe haemodynamic effects. Lab Anim 38:64-69.

Tamamaki N, Yanagawa Y, Tomioka R, Miyazaki JI, Obata K, Kaneko T (2003) Green fluorescent protein expression and colocalization with calretinin, parvalbumin, and somatostatin in the GAD67-GFP knock-in mouse. J Comp Neurol 467:60-79.

Toney GM, Porter JP (1993) Functional role of brain AT1 and AT2 receptors in the central angiotensin II pressor response. Brain Res 603:57-63.

Tucker DC, Saper CB (1985) Specificity of spinal projections from hypothalamic and brainstem areas which innervate sympathetic preganglionic neurons. Brain Res 360:159-164.

Wamsteeker Cusulin J, Füzesi T, Watts A, Bains J (2013) Characterization of corticotropin-releasing hormone neurons in the paraventricular nucleus of the hypothalamus of Crh-IRES-Cre mutant mice. PLoS One 8:e64943.

Yosten GL, Pate AT, Samson WK (2011) Neuronostatin acts in brain to biphasically increase mean arterial pressure through sympatho-activation followed by vasopressin secretion: the role of melanocortin receptors. Am J Physiol Regul Integr Comp Physiol 300:R1194-R1199.

Zhu Q, Guo SY, Gong S, Yin QZ, Hisamitsu T, Jiang XH (2005) Losartan blocks the excitatory effect of peripheral hypertonic stimulation on vasopressinergic neurons in hypothalamic paraventricular nucleus in rats: electrophysiological and immunocytochemical evidence. Neurosci Lett 380:12-16. 